\title{
Exponential Convergence through Linear Finite Element Discretization of Stratified Subdomains
}

\author{
Murthy N. Guddati ${ }^{1^{*}}$, Vladimir Druskin ${ }^{2}$, Ali Vaziri Astaneh ${ }^{1}$ \\ ${ }^{1}$ Department of Civil Engineering, North Carolina State University, Raleigh, NC 27695-7908, USA \\ ${ }^{2}$ Schlumberger Doll Research, Cambridge, MA 02139, USA
}

\begin{abstract}
Motivated by problems where the response is needed at select localized regions in a large computational domain, we devise a novel finite element discretization that results in exponential convergence at pre-selected points. The key features of the discretization are (a) use of midpoint integration to evaluate the contribution matrices, and (b) an unconventional mapping of the mesh into complex space. Named complex-length finite element method (CFEM), the technique is linked to Padé approximants that provide exponential convergence of the Dirichlet-to-Neumann maps and thus the solution at specified points in the domain. Exponential convergence facilitates drastic reduction in the number of elements. This, combined with sparse computation associated with linear finite elements, results in significant reduction in the computational cost. The paper presents the basic ideas of the method as well as illustration of its effectiveness for a variety of problems involving Laplace, Helmholtz and elastodynamics equations.
\end{abstract}

Keywords Optimal grids, Padé approximants, Dirichlet-to-Neumann maps, Rational approximation, Spectral element methods

\section{Introduction}

Conventional domain-based methods such as finite element and finite difference techniques obtain the solution over the entire domain. While such approaches are appropriate for many problems, there are several situations where the response is needed only in a few small regions of interest. Some examples include: (a) reservoir modeling where the response at injection and production wells are of utmost

\footnotetext{
* Corresponding author. Tel.: +1 919515 7699; fax: +1 9195157908

E-mail address: murthy.guddati@ncsu.edu
} 
importance, (b) forward modeling in the context of nondestructive testing and system identification, where the goal is to match the response of the system at sparse discrete points in the domain and (c) structural acoustics, where the acoustic signature is not needed at all the points, but at distinct locations in the far field. Most of these problems involve significant computational expense and it would be desirable to reduce the computational cost, if it can come at the expense of not computing the response in the rest of the domain. With this motivation, this paper presents an unconventional finite element method that provides high accuracy at prescribed points in the domain. In this paper, we treat the special but important class of problems involving large regular (e.g. layered) subdomains, where the actual solution inside these subdomains may not be of interest, but it is important to capture the effect of these subdomains on the solution in the remainder of domain. Specifically, we show that a special mesh with midpoint-integrated linear finite elements results in exponential convergence of the solution on the edges of layered subdomains.

Exponential convergence is typically achieved with the help of spectral methods where the field variable is discretized with Fourier basis $[1,2]$, but these methods typically render the computation global. On the other hand, regular finite element and finite difference methods involve more efficient sparse computation, but the convergence is only algebraic. It was discovered that exponential convergence can be obtained with sparse computation, provided that the solution is needed only at specific points in the domain [3, 4]. By linking finite-difference approximation to rational approximation of the Dirichlet-to-Neumann (DtN) map, exponential convergence is achieved at the edges of subdomains discretized with specially devised finite difference grids. The basic idea is to obtain optimal rational approximation of the one-sided DtN map (with Dirichlet or Neumann condition applied on the other edge), and translate the approximation to an equivalent finite difference grid. Since the grids result from exponentially convergent optimal approximations of the DtN map, they are called optimal finite difference grids and result in exponential convergence at the edges of the sub-domains. The main limitation of this method is that two distinct finite difference grids are needed, one when Dirichlet condition is applied on the other edge, and another for Neumann condition, indicating that the grids cannot be used directly for two-sided problems, which would be the building block for multi-domain problems. Optimal grids can be used for two-sided problems indirectly, through splitting the solution into odd and even parts, devising two separate grids for each part, and using them in a completely overlapping fashion. This idea is extended to multiple dimensions, but the computation becomes rather cumbersome, requiring increasing number of overlapping grids (four for two-dimensional problems and eight for threedimensions) [4-6].

In this paper, we introduce a simpler alternative to optimal finite difference grids and eliminate the need for overlapping grids. The key to the proposed method is the observation that midpoint integrated 
linear finite elements preserve the DtN map of the half-space, i.e. adding these elements to a half-space does not alter the DtN map of the composite half-space [7-9]; given the equivalence between impedance and half-space DtN map, we call this property the impedance-preserving property. We show that this property eliminates the need for multiple overlapping grids and provides exponential convergence of the DtN map for the two-sided problem with a single grid. This makes the implementation attractive and the computation can be performed by a simple modification of existing finite element codes. The only complication is that the finite element mesh needs to be bent out of the real space, making the element lengths complex-valued. This feature necessitates complex arithmetic and could contribute to an increase in the computational cost. However, this increase is not an issue as the proposed method needs a very coarse grid, with number of elements typically less by an order of magnitude than that for regular finite element discretization. Moreover, in many cases including time-harmonic wave propagation, the original computation involves complex arithmetic and the mapping of the mesh into complex space does not add any further complications.

This paper focuses on the derivation of the method along with numerical examples to illustrate its efficiency. We start with the two-dimensional model problem given in Fig. 1 and show that it can be reduced to a set of one-dimensional two-point boundary value problems through semi-discretization in the vertical direction (Section 2). We then obtain a grid that simultaneously approximates the DtN map for all of the one-dimensional problems (Section 3). This is achieved by reformulating midpoint integrated linear finite elements, described in Section 3.1, as Crank-Nicolson discretization of an equivalent first-order system (Section 3.2). Exponential convergence is achieved by choosing the parameters by linking the Crank-Nicolson discretization with Padé approximants (Section 3.3). The result is a finite element mesh with complex element lengths, as discussed in Section 3.3. In Section 4 we show the applicability of the present method to general vector (elastic) equations. Section 5 contains numerical examples illustrating the exponential convergence and practical use of the proposed method.

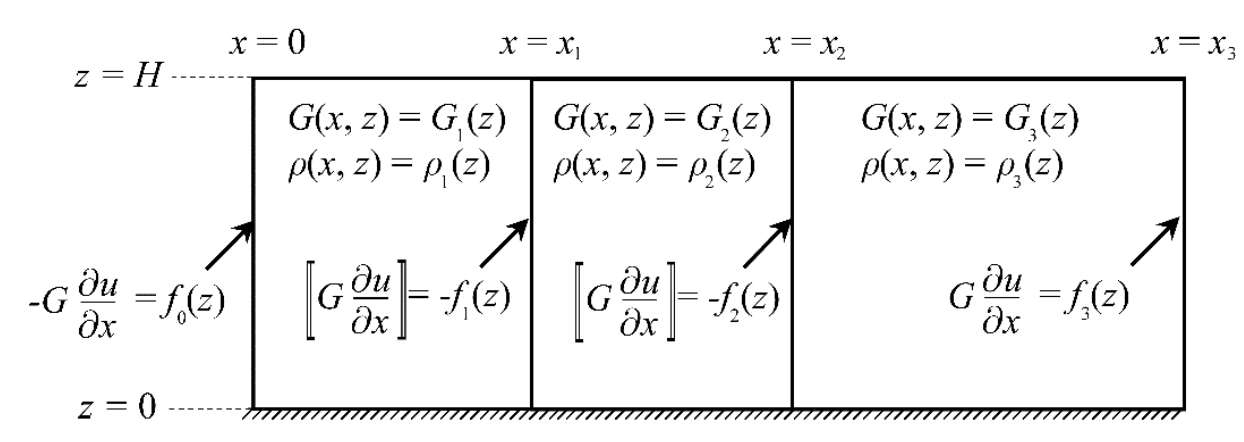

Fig. 1. A schematic of the model problem. An elastic layer is deforming out of plane under external excitation $f_{0,1,2,3}$ at the vertical interfaces. The segments bounded by these interfaces can be stratified in the vertical direction. The goal is to obtain the responses at the interfaces. 


\section{Preliminaries}

\subsection{Problem statement}

For the sake of focused discussion, consider the model problem in Fig. 1, where an elastic layer with three segments, each individually stratified in the vertical direction. Excitation is applied only at the vertical edges and interfaces, and the goal is to obtain the response at these locations. We consider the anti-plane shear deformation governed by the Helmholtz equation (Laplace equation being a special case when frequency $\omega=0)$ :

$$
-\frac{\partial}{\partial z}\left(G(x, z) \frac{\partial w}{\partial z}\right)-\frac{\partial}{\partial x}\left(G(x, z) \frac{\partial w}{\partial x}\right)-\rho(x, z) \omega^{2} w=0,
$$

where $w$ is the anti-plane deformation, $G$ is the shear modulus and $\rho$ is the density. Since the forces are applied only at the vertical interfaces and the response is needed only at these locations, it is sufficient to solve the coupled problem obtained by assembling the Dirichlet-to-Neumann (DtN) maps, of individual segments. We thus focus on obtaining an accurate estimate of the DtN map for a single segment, which reduces the problem to:

$$
\text { Problem 1: }\left\{\begin{array}{l}
\text { Obtain the relationship DtN: }\left.\left.w\right|_{x=0, L} \rightarrow \frac{d w}{d x}\right|_{x=0, L}, \text { with } w \text { satisfying: } \\
-\frac{\partial}{\partial z}\left(G(z) \frac{\partial w}{\partial z}\right)-\frac{\partial}{\partial x}\left(G(z) \frac{\partial w}{\partial x}\right)-\rho(z) \omega^{2} w=0,
\end{array}\right.
$$

\subsection{Dimensional reduction}

Our ultimate goal is to obtain a finite element mesh that can yield accurate results at the vertical interfaces. Assuming that accuracy is needed along the entire length of every interface, we perform $z$ discretization in a conventional manner and independent of $x$ coordinate. It is also natural to make $x$ discretization independent of $z$, resulting in a two-dimensional tensor-product mesh. For the purposes of analyzing the method, the tensor product discretization can be viewed as discretization in $z$ followed by discretization in $x$. In this subsection, we focus on the discretization in $z$ and the resulting dimensionally reduced differential equation in $x$.

Dimensional reduction is performed by (a) multiplying the governing equation (2) by virtual 
displacement by $\delta w$, (b) performing integration by parts in $z$, and (c) applying the boundary conditions at $z=0, H$, resulting in:

$$
\int_{z=0}^{z=L}\left(\frac{\partial \delta w}{\partial z} G(z) \frac{\partial w}{\partial z}+\delta w G(z) \frac{\partial^{2} w}{\partial x^{2}}-\delta w \omega^{2} \rho(z) w\right) d z=0 \text { for all } \delta w, \quad 0<x<L .
$$

We apply Bubnov-Galerkin method, i.e. use the following approximations,

$$
w \approx \mathbf{N}_{z}(z) \mathbf{W}(x) \text { and } \delta w \approx \mathbf{N}_{z}(z) \delta \mathbf{W}(x),
$$

where $\mathbf{N}_{z}$ is the matrix of interpolation functions (shape function matrix), and $\mathbf{W}$ and $\delta \mathbf{W}$ are vectors of discretized displacement and virtual displacement respectively. We emphasize that $\mathbf{W}$ and $\delta \mathbf{W}$ are functions of $x$ as the discretization is performed only in the $z$ direction. After substituting (4) in (3) and performing appropriate manipulations, we obtain the discrete form of the governing equation:

$$
\mathbf{R W}-\mathbf{H} \frac{d^{2} \mathbf{W}}{d x^{2}}-\omega^{2} \mathbf{M W}=\mathbf{0}, \text { for } 0<x<L,
$$

where,

$$
\mathbf{R}=\int_{z=0}^{z=H} \frac{\partial \mathbf{N}_{z}{ }^{T}}{\partial z} G \frac{\partial \mathbf{N}_{z}}{\partial z} d z, \quad \mathbf{H}=\int_{z=0}^{z=H} \mathbf{N}_{z}^{T} G \mathbf{N}_{z} d z, \quad \mathbf{M}=\int_{z=0}^{z=H} \mathbf{N}_{z}^{T} \rho \mathbf{N}_{z} d z .
$$

Equation (5) can be decoupled into a set of differential equations through eigen-decomposition:

$$
-\frac{\partial^{2} u_{l}}{\partial x^{2}}+\lambda_{l} u_{l}=0 \quad \text { for } 0<x<L, \quad \lambda_{\min } \leq \lambda_{l} \leq \lambda_{\max },
$$

where $\lambda_{l}$ are the eigenvalues of $\mathbf{H}$ with respect to $\mathbf{R}-\omega^{2} \mathbf{M} . u_{l}$ are the weights of the corresponding eigenvectors (modal participation factors). Note that $\lambda_{l}$ are real and bounded because all the matrices are symmetric and $\mathbf{A}$ is positive definite.

With the above decoupling, the original problem of obtaining the two-dimensional DtN map simplifies to finding the DtN maps for a set of one-dimensional boundary value problems:

$$
\text { Problem 2: }\left\{\begin{array}{l}
\text { Obtain the relationship DtN: }\left.\left.u\right|_{x=0, L} \rightarrow \frac{d u}{d x}\right|_{x=0, L} \text {, with } u \text { satisfying: } \\
-\frac{\partial^{2} u}{\partial x^{2}}+\lambda u=0 \text { for } 0<x<L, \quad \lambda_{\min } \leq \lambda \leq \lambda_{\max } .
\end{array}\right.
$$

Note that the subscript $l$ is not shown this point forward for simplicity in presentation. For the special case of homogeneous media, the shape functions $\mathbf{N}_{z}$ can be chosen according to Fourier expansion, directly resulting in decoupled system of equations in (7). Nevertheless, we use general discretization in the $z$ direction, to ensure applicability to stratified media. Note that eigen-decomposition leading to (7) is performed only for the purposes of analyzing the method; it is never performed in the actual computation. 


\subsection{A closer look at the DtN map}

The desired DtN map in (8) is a $2 \times 2$ matrix,

$$
\left\{\begin{array}{c}
-v_{0} \\
v_{L}
\end{array}\right\}=\underbrace{\left[\begin{array}{ll}
K_{00} & K_{0 L} \\
K_{L 0} & K_{L L}
\end{array}\right]}_{=\mathbf{K}}\left\{\begin{array}{l}
u_{0} \\
u_{L}
\end{array}\right\},
$$

where $v=\partial u / \partial x$ and the subscripts represent the locations at which the quantities are evaluated. The negative sign for $v_{0}$ is because the outside normal at $x=0$ is in the negative $x$ direction. Considering the geometric symmetry of the domain as well as the symmetry of the operator, the DtN map takes the form,

$$
\mathbf{K}=\left[\begin{array}{cc}
K_{\text {diag }} & K_{\text {off }} \\
K_{\text {off }} & K_{\text {diag }}
\end{array}\right] \text {. }
$$

For a fixed $\lambda$, the exact DtN map can be easily obtained by solving the two-point boundary value problem in (8), and is given by,

$$
\mathbf{K}_{\text {exact }}=\left[\begin{array}{ll}
K_{\text {diag }} & K_{\text {off }} \\
K_{\text {off }} & K_{\text {diag }}
\end{array}\right]=\frac{\sqrt{\lambda}}{\sinh (\sqrt{\lambda} L)}\left[\begin{array}{cc}
\cosh (\sqrt{\lambda} L) & -1 \\
-1 & \cosh (\sqrt{\lambda} L)
\end{array}\right] .
$$

Note that the exact DtN map satisfies the impedance-preserving property, defined as follows: when $\mathbf{K}_{\text {exact }}$ is augmented with the exact DtN map of a half-space $\left(\mathbf{K}_{\text {halspace }}=\sqrt{\lambda}\right)$ and the interior node is eliminated, exact half-space stiffness (and thus impedance) is preserved at the exterior node (this follows from the simple physical argument that when a layer is added to a half-space with same material properties, we obtain the same half-space). In other words, impedance preserving property states that, when a layer $\left(x_{0}, x_{1}\right)$ is augmented with a half-space $\left(x_{1}, \infty\right)$, the Neumann data required at $x_{0}$ to generate a Dirichlet data of $u_{0}$ is $K_{\text {halspace }} u_{0}$, i.e.,

$$
\left[\begin{array}{cc}
K_{\text {diag }} & K_{\text {off }} \\
K_{\text {off }} & K_{\text {diag }}+K_{\text {halfspace }}
\end{array}\right]\left\{\begin{array}{l}
u_{0} \\
u_{1}
\end{array}\right\}=\left\{\begin{array}{c}
K_{\text {halfspace }} u_{0} \\
0
\end{array}\right\} .
$$

It is easy to show that the above definition is equivalent to,

$$
K_{\text {halfspace }}=K_{\text {diag }}-\frac{K_{\text {off }}^{2}}{\left(K_{\text {diag }}+K_{\text {halfspace }}\right)} .
$$

Noting that $K_{\text {halspace }}=\sqrt{\lambda}$, it is easy to see that impedance-preserving property takes the simple form,

$$
K_{\text {diag }}^{2}-K_{\text {off }}^{2}=\lambda
$$

The exact DtN map in (11), while useful for any given $\lambda$, is not efficient for solving the original 2D 
problem, as it requires expensive eigen-decomposition and associated transformations. A more efficient approach of solving the 2D problem would be to devise a grid that simultaneously works for all $\lambda$. One option is to use the standard equidistant finite element or finite difference grid; such as grid results in algebraic convergence of the solution over the entire domain, and thus the DtN map. Since our goal involves accurate approximation of just the DtN map, it may be worth obtaining higher accuracy for the DtN map, possibly at the expense of the accuracy in the interior solution. Druskin and coworkers [3, 4, 10-12] have successfully utilized this idea to obtain exponential convergence of one-sided DtN map, i.e., the DtN map at one end of the domain with the specified boundary condition at the other end. Utilizing rational approximation theory of Stieltjes functions and its link to staggered-grid finite difference method, they obtained two independent grids that provide exponential convergence of two one-sided DtN maps, one for Dirichlet boundary condition at the other end, and the other for Neumann boundary condition. They also extended the method to the two-sided problem, but the extension is not straightforward. They split the solution into odd and even parts and use two separate grids to approximate the corresponding DtN maps. Simultaneous solution of two-point boundary value problem requires the use of these grids in a completely overlapping fashion, making the implementation cumbersome, especially when extended to higher dimensions (we would need to use four overlapping grids for 2D and eight for 3D) [4-6]. Therefore, it is desirable to obtain a single mesh that would simultaneously approximate all the elements of the $2 \times 2 \mathrm{DtN}$ maps with equal accuracy. As shown in the rest of the paper, the midpoint-integrated linear finite elements proposed by Guddati and coworkers [7-9] leads to such approximation. The key is that these elements satisfy the impedance-preserving property in (14). We utilize this property and develop linear finite element discretization that provides simultaneous exponential convergence for both diagonal and off-diagonal elements of the DtN map. The resulting finite element mesh is not a standard discretization of the real domain $(0, L)$, but a mesh mapped into the complex space (the element lengths are complex-valued). We thus term this the Complex-length Finite Element Method (CFEM); the remainder of the paper contains the formulation of CFEM and the illustration of its effectiveness.

\section{Complex-length finite element method (CFEM)}

\subsection{Linear finite elements with midpoint integration}

Consider the domain $(0, L)$ discretized into non-overlapping finite elements of lengths $L_{j}, j=1, \cdots, n$, $\sum L_{j}=L$, with nodes located at $x_{0}, x_{1}, \ldots, x_{n}$. Restricting the discussion to the $j$ th element, the weak form becomes, 


$$
\int_{x_{j-1}}^{x_{j}}\left(\frac{\partial \delta u}{\partial x} \frac{\partial u}{\partial x}+\delta u \lambda u\right) d x=\delta u_{j} v_{j}-\delta u_{j-1} v_{j-1}
$$

where $\delta u$ is the variation of $u$. Utilizing Bubnov-Galerkin method with linear interpolation within the element and after performing the transformation $\bar{x}=x-x_{j-1}$, we obtain,

$$
\left\{\begin{array}{c}
\delta u_{j-1} \\
\delta u_{j}
\end{array}\right\}^{T}\left[\int_{0}^{L_{j}}\left(\left\{\begin{array}{c}
-1 / L_{j} \\
1 / L_{j}
\end{array}\right\}\left\{\begin{array}{c}
-1 / L_{j} \\
1 / L_{j}
\end{array}\right\}^{T}+\left\{\begin{array}{c}
1-\bar{x} / L_{j} \\
\bar{x} / L_{j}
\end{array}\right\} \lambda\left\{\begin{array}{c}
1-\bar{x} / L_{j} \\
\bar{x} / L_{j}
\end{array}\right\}\right) d \bar{x}\right]\left\{\begin{array}{c}
u_{j-1} \\
u_{j}
\end{array}\right\}=\left\{\begin{array}{c}
\delta u_{j-1} \\
\delta u_{j}
\end{array}\right\}^{T}\left\{\begin{array}{c}
-v_{j-1} \\
v_{j}
\end{array}\right\} .
$$

The next step is to evaluate the integral using the midpoint rule. Performing such integration and eliminating the virtual displacements, we obtain the DtN map (stiffness relation) for a single element:

$$
\left\{\begin{array}{c}
-v_{j-1} \\
v_{j}
\end{array}\right\}=\left[\begin{array}{cc}
\left(\frac{1}{L_{j}}+\frac{\lambda L_{j}}{4}\right) & \left(-\frac{1}{L_{j}}+\frac{\lambda L_{j}}{4}\right) \\
\left(-\frac{1}{L_{j}}+\frac{\lambda L_{j}}{4}\right) & \left(\frac{1}{L_{j}}+\frac{\lambda L_{j}}{4}\right)
\end{array}\right]\left\{\begin{array}{c}
u_{j-1} \\
u_{j}
\end{array}\right\} .
$$

In the above DtN map, $K_{\text {diag }}^{2}-K_{\text {off }}^{2}=\lambda$, which indicates the impedance-preserving property (see (14)). Since all the finite elements in the mesh satisfy this property, the two-point DtN map of the entire mesh would also satisfy the impedance-preserving property. An important consequence is that, a mesh that results in exponential convergence of the diagonal element of the DtN map, also results in exponential convergence of the off-diagonal element (this is because, $K_{\text {diag, approx }}^{2}-K_{\text {off, approx }}^{2}=\lambda=K_{\text {diag, exact }}^{2}-K_{\text {off, exact }}^{2}$ ), and thus exponential convergence of the entire two-sided DtN map.

To obtain the DtN map of the discretized domain, we could assemble the $2 \times 2$ DtN maps of the individual elements and eliminate the interior degrees of freedom by taking Schur complement. This process renders the computation global, making it cumbersome to analyze. To facilitate easier analysis, we turn to an equivalent propagator matrix approach, by converting the boundary value problem into an initial value problem.

\subsection{An alternative view: first-order form}

We can verify that the DtN relation in (17) is equivalent to,

$$
\left\{\begin{array}{c}
\frac{u_{j}-u_{j-1}}{L_{j}} \\
\frac{\bar{v}_{j}-\bar{v}_{j-1}}{L_{j}}
\end{array}\right\}=\left[\begin{array}{cc}
0 & \sqrt{\lambda} \\
\sqrt{\lambda} & 0
\end{array}\right]\left\{\begin{array}{l}
\frac{u_{j}+u_{j-1}}{2} \\
\frac{\bar{v}_{j}+\bar{v}_{j-1}}{2}
\end{array}\right\}
$$


where $\bar{v}=v / \sqrt{\lambda}$. It can be immediately seen that the above equation is the Crank-Nicolson discretization of first order form of differential equation (7), i.e.,

$$
\frac{\partial}{\partial x}\left\{\begin{array}{l}
u \\
\bar{v}
\end{array}\right\}=\left[\begin{array}{cc}
0 & \sqrt{\lambda} \\
\sqrt{\lambda} & 0
\end{array}\right]\left\{\begin{array}{l}
u \\
\bar{v}
\end{array}\right\} \text { for } 0<x<L, \quad \lambda_{\min } \leq \lambda \leq \lambda_{\max } .
$$

Equation (18) can also be written in the form,

$$
\left\{\begin{array}{l}
u_{j} \\
\bar{v}_{j}
\end{array}\right\}=\mathbf{P}_{j}\left\{\begin{array}{l}
u_{j-1} \\
\bar{v}_{j-1}
\end{array}\right\}
$$

where $\mathbf{P}_{j}$ is the propagator matrix associated with the finite element, which is inherently connected to its DtN map in (17). The advantage of the propagator matrix approach is that the propagator matrix of the complete interval $(0, L), \mathbf{P}$, is obtained by simply multiplying the propagator matrices of individual finite elements:

$$
\left\{\begin{array}{l}
u_{L} \\
\bar{v}_{L}
\end{array}\right\}=\mathbf{P}_{n}\left\{\begin{array}{l}
u_{n-1} \\
\bar{v}_{n-1}
\end{array}\right\}=\mathbf{P}_{n} \mathbf{P}_{n-1}\left\{\begin{array}{l}
u_{n-2} \\
\bar{v}_{n-2}
\end{array}\right\}=\cdots=\underbrace{\mathbf{P}_{n} \mathbf{P}_{n-1} \cdots \mathbf{P}_{2} \mathbf{P}_{1}}_{\mathbf{P}}\left\{\begin{array}{l}
u_{0} \\
\bar{v}_{0}
\end{array}\right\} .
$$

The matrix $\mathbf{P}$ can also be written in the form of DtN relation in (9), implying that successful approximation of the propagator matrix would automatically result in successful approximation of the DtN map. Thus, the problem reduces to, finding the mesh parameters $L_{j}$ that would result in exponential convergence of the propagator matrix $\mathbf{P}$ for various values of $\lambda$ on the real line.

The problem of approximating the propagator matrix can be further simplified by decoupling the system of equations in (19). Through eigen-decomposition, we have,

$$
\frac{\partial}{\partial x}\left\{\begin{array}{l}
\psi_{1} \\
\psi_{2}
\end{array}\right\}=\left[\begin{array}{cc}
+\sqrt{\lambda} & 0 \\
0 & -\sqrt{\lambda}
\end{array}\right]\left\{\begin{array}{l}
\psi_{1} \\
\psi_{2}
\end{array}\right\} \text { for } 0<x<L, \quad \lambda_{\min } \leq \lambda \leq \lambda_{\max },
$$

where the diagonal matrix contains the eigenvalues of the operator in (19), and $\psi_{1}, \psi_{2}$ are the weights associated with the corresponding eigenvectors. Thus the DtN approximation problem reduces to:

$$
\text { Problem 3: }\left\{\begin{array}{l}
\text { Find the mesh parameters } L_{j} \text { that would result in exponentially } \\
\text { convergent propagator matrix for the interval }(0, L) \text { for the equation: } \\
\frac{\partial \psi}{\partial x}=\alpha \psi \text { for all } \alpha \in\left(-\sqrt{\left|\lambda_{\max }\right|}, \sqrt{\left|\lambda_{\max }\right|}\right) \cup\left(-i \sqrt{\left|\lambda_{\min }\right|}, i \sqrt{\left|\lambda_{\min }\right|}\right) .
\end{array}\right.
$$

In the above, $\alpha=\sqrt{\lambda}$ and $\lambda_{\min }$ is implicitly assumed to be negative, indicating that $\alpha$ could be imaginary. It is instructive to note the physical meaning of the eigenvectors associated with the decoupled form in (22). The eigenvector associated with $\psi_{1}$ is $\left\{\begin{array}{ll}u & \bar{v}\end{array}\right\} \propto\left\{\begin{array}{ll}1 & 1\end{array}\right\}$, or equivalently, $\bar{v}=u$, which 
corresponds to an exponentially growing solution, $u=\psi_{1} e^{\sqrt{x} x}$. The other eigenvector is $\left\{\begin{array}{ll}u & \bar{v}\end{array}\right\} \propto\left\{\begin{array}{ll}1 & -1\end{array}\right\}$, which corresponds to a decaying solution, $u=\psi_{2} e^{-\sqrt{\lambda} x}$. These are the solutions of the original second order differential equation (7). This point of view is important as our method is based on approximation of the exponential functions (see Section 3.3).

The impedance-preserving property of the midpoint-integrated linear finite elements can be proven more elegantly as follows. The impedance relation for the half-space $(0, \infty)$ is: $\partial u /\left.\partial x\right|_{x=0}=\sqrt{\lambda} u_{0}$, or $\bar{v}_{0}=u_{0}$ (this corresponds to the decaying solution). The impedance-preserving property can be stated as: exact impedance relation $\bar{v}=u$ at $x=0$ implies $\bar{v}=u$ at any $x$. The impedance-preserving property of the exact propagator can be illustrated as follows: Equation (19) is invariant to the swapping of $u$ with $\bar{v}$; therefore, if the initial condition at $x=0$ is swap-invariant $(\bar{v}=u)$, the "final" condition at any $x$ must also be swap-invariant $(\bar{v}=u)$. Fortunately, the swap-invariance of (19) is preserved through the discretization in (18), implying that the Crank-Nicolson propagator, and thus the midpoint-integrated finite element, satisfy the impedance-preserving property.

\subsection{Selection of the mesh parameters}

Considering that the exact solution to (23) is of the form $A e^{\alpha x}$, the propagator associated with the interval $(0, L)$ is simply,

$$
P_{\text {exact }}=\frac{\psi_{L}}{\psi_{0}}=e^{\alpha L} .
$$

The approximate propagator for the $j$ th interval is given by the Crank-Nicolson method,

$$
\frac{\psi_{j}-\psi_{j-1}}{L_{j}}=\alpha\left(\frac{\psi_{j}+\psi_{j-1}}{2}\right) \Rightarrow \psi_{j}=\underbrace{\left(\frac{1+\alpha L_{j} / 2}{1-\alpha L_{j} / 2}\right)}_{=P_{j}} \psi_{j-1} .
$$

The propagator for the entire interval $(0, L)$ is the product of all the propagators, i.e.,

$$
P_{\text {approx }}=\prod_{j=1}^{n} P_{j}=\prod_{j=1}^{n}\left(\frac{1+\alpha L_{j} / 2}{1-\alpha L_{j} / 2}\right) .
$$

Note that $P_{\text {approx }}$ is a so-called relative approximant of $e^{\alpha L}$, which is a rational function of the form $Q(\alpha) / Q(-\alpha)$, where $Q$ is a polynomial of degree $n$ with roots $-2 / L_{j}$. Relative approximants are wellstudied subject of rational approximation theory [13], and there is a variety of approximants convergent 
on the real axis at least exponentially with respect to $n$. Obviously, $Q(\alpha) / Q(-\alpha)$ must not have poles and residues on the real axis in order to be a good approximant of the exponent there; this is the reason why $L_{j}$ must be complex, implying that the mesh has to be mapped into the complex space. Hence the current method is called the Complex-length Finite Element Method (CFEM).

In this paper, we consider the relative (diagonal) Padé approximant matching the first $2 n$ terms of Taylor expansion at $\alpha=0$. In other words, the 0 to $2 n^{\text {th }}$ derivatives of the approximant must match with that of the exact solution at $\alpha=0$, i.e.,

$$
\left.\frac{d^{j} P_{p a d e}}{d \alpha^{j}}\right|_{\alpha=0}=\left.\frac{d^{j}\left(e^{\alpha L}\right)}{d \alpha^{j}}\right|_{\alpha=0}, \quad j=0, \cdots, 2 n .
$$

The reason for the choice of Padé approximant is its simplicity. There are a number of approximants of the form $Q(\alpha) / Q(-\alpha)$ with better convergence properties for larger $|\alpha|$; we plan to consider such approximations in future research.

For the Padé approximant considered in (27), the roots $-2 / L_{j}$ and thus the element lengths $L_{j}$ can be computed with a standard algorithms (see e.g. [14]). The resulting mesh has the following properties:

1. $L_{j}$ are independent of $\alpha$ (or $\lambda$ ), indicating that the same mesh can be used for the range of complex $\lambda$ given in (8), implying that the mesh is applicable to the original 2D problem in (2).

2. $L_{j}$ come in complex conjugate pairs. It follows from the fact that the Padé approximant is real for any real $\alpha$.

3. $\sum L_{j}=L$ follows directly from evaluating (27) for $j=1$, which indicates that the end points match with the physical edges of the domain. This is an important property since the procedure for the subdomain does not affect the geometry of the rest of the analysis domain.

4. A peculiar property of midpoint-integrated finite element grid is that the DtN map is invariant of the ordering of the elements. This implies that the mesh is not unique; we order the mesh so that it is symmetric and smooth (as smooth as possible) in the complex plane. Such a mesh is obtained by ordering the elements with increasing or decreasing phase angle of their lengths (see Section 5.2).

5. The mesh scales with the length of the domain and we can tabulate the element sizes relative to the domain size, i.e. $L_{j} / L$, for any given number of elements; this is done in Table 1 up to 16 elements. In general one can obtain $L_{j} / L=2 / x_{j}$ where $x_{j}$ are the roots of the $n^{\text {th }}$-degree polynomial (see e.g. [14]): 


$$
\sum_{j=0}^{n} \frac{(2 n-j) !}{j !(n-j) !}(-x)^{j}=0
$$

Following the order suggested in item 4 above, the meshes are shown Fig. 2. Meshes with odd and even number of elements are shown in separate figures for clarity in presentation. With refinement, the mesh appears to converge to a specific curve in the complex space. We suspect that there is an analytical form for the converged mesh, linked to the asymptotic behavior of the poles of Padé approximants, similar to the one known for optimal approximants on $[0, \infty)[15]$.

6. It is known that the real parts of the roots $\left(-2 / L_{j}\right)$ are positive for the diagonal Padé approximants of exponential function [14, 16], implying that the real parts of $L_{j}$ are positive and the mesh is not bent outside the domain (or have any knots), which is another physically appealing aspect.

\subsection{Summary: CFEM discretization procedure and additional properties}

We suggest the following approach for CFEM discretization of the model problem in Fig. 1:

1. Divide the problem into multiple subdomains bounded by points where (a) the material properties are discontinuous, (b) loads are applied, or (c) the solution is desired.

2. Discretize each sub-domain using CFEM mesh as described in the previous subsection.

3. Combine all the sub-domain grids and perform finite element analysis on the entire domain. Utilize midpoint integration to evaluate the contribution matrices.

4. Extract the results from the sub-domain interfaces. Note that the displacements in the interior of the sub-domains have no immediate physical meaning.

Table 1 Element lengths for varying number of elements $(n)$. The domain size is unity. For other cases, the element lengths simply scale with domain size.

\begin{tabular}{cc|cc}
\hline$n=1$ & 1.00000000000000 & $n=2$ & $0.50000000000000 \pm 0.28867513459481 i$ \\
\hline \multirow{2}{*}{$n=3$} & $0.28468557688388 \pm 0.27159985141630 i$ & & $0.18313248053143 \pm 0.23132522602625 i$ \\
& 0.43062884623222 & $n=4$ & $0.31686751946856 \pm 0.09488202514221 i$ \\
\hline \multirow{2}{*}{$n=5$} & $0.12803667831541 \pm 0.19668213834621 i$ & & $0.09489061789607 \pm 0.16944514819433 i$ \\
& $0.23485450871940 \pm 0.12209940763707 i$ & $n=6$ & $0.17914640739749 \pm 0.12594324946340 i$ \\
& 0.27421762593037 & & $0.22596297470643 \pm 0.04614135671779 i$ \\
\hline
\end{tabular}




\begin{tabular}{|c|c|c|c|}
\hline$n=7$ & $\begin{array}{c}0.07338559568636 \pm 0.14811940741461 i \\
0.14065739395847 \pm 0.12154781833235 i \\
0.18538954553266 \pm 0.06776497788782 i \\
0.20113492964499\end{array}$ & $n=8$ & $\begin{array}{l}0.05861791492234 \pm 0.13119236974564 i \\
0.11325833004971 \pm 0.11445496413908 i \\
0.15337794771885 \pm 0.07709430353886 i \\
0.17474580730908 \pm 0.02713226173787 i\end{array}$ \\
\hline$n=9$ & $\begin{array}{c}0.04802049907890 \pm 0.11752570488080 i \\
0.09316287173966 \pm 0.10679717990370 i \\
0.12840441052931 \pm 0.08014721079992 i \\
0.15100957026047 \pm 0.04281546509628 i \\
0.158805296783284\end{array}$ & $n=10$ & $\begin{array}{l}0.04014472910062 \pm 0.10630697796687 i \\
0.07802273616547 \pm 0.09940003581225 i \\
0.10881874816902 \pm 0.07996015060428 i \\
0.13078256453612 \pm 0.05163001172938 i \\
0.14223122202876 \pm 0.01782841382104 i\end{array}$ \\
\hline$n=11$ & $\begin{array}{c}0.03412261657800 \pm 0.09695789626293 i \\
0.06634486381072 \pm 0.09256005182226 i \\
0.09329088025857 \pm 0.07811366645707 i \\
0.11386072467713 \pm 0.05628616844255 i \\
0.12678425930221 \pm 0.02942684799389 i \\
0.131193310746699\end{array}$ & $n=12$ & $\begin{array}{l}0.02940803944815 \pm 0.08906181395662 i \\
0.05715192456673 \pm 0.08635352530097 i \\
0.08082582076929 \pm 0.07545289071966 i \\
0.09976290741316 \pm 0.05839885662872 i \\
0.11301395814524 \pm 0.03687019624343 i \\
0.11983734965741 \pm 0.01259866487095 i\end{array}$ \\
\hline$n=13$ & $\begin{array}{c}0.02564318775369 \pm 0.08231355321087 i \\
0.04978573946440 \pm 0.08076582096948 i \\
0.07069390925651 \pm 0.07243833050796 i \\
0.08799247146698 \pm 0.05894563008011 i \\
0.10097469339377 \pm 0.04152986688324 i \\
0.10902977699627 \pm 0.02144314210934 i \\
0.11176044333671\end{array}$ & $n=14$ & $\begin{array}{l}0.02258550311646 \pm 0.07648569373967 i \\
0.04379127631258 \pm 0.07574740948845 i \\
0.06236027779351 \pm 0.06932300610809 i \\
0.07811546938314 \pm 0.05852853926583 i \\
0.09053178744825 \pm 0.04431195125450 i \\
0.09911464460927 \pm 0.02760255210502 i \\
0.10350104133675 \pm 0.00937153174338 i\end{array}$ \\
\hline$n=15$ & $\begin{array}{c}0.02006570730347 \pm 0.07140591920396 i \\
0.03884638966705 \pm 0.07123849065223 i \\
0.05542991160544 \pm 0.06624511011050 i \\
0.06977487505357 \pm 0.05752432367232 i \\
0.08149231310591 \pm 0.04581962272804 i \\
0.09018490369015 \pm 0.03183750267162 i \\
0.09553514496841 \pm 0.01630985840134 i \\
0.09734150921194\end{array}$ & $n=16$ & $\begin{array}{l}0.01796266496341 \pm 0.06694162366630 i \\
0.03471809507502 \pm 0.06717964470869 i \\
0.04960794930403 \pm 0.06327803486714 i \\
0.06268398209122 \pm 0.05617188543383 i \\
0.07365957371625 \pm 0.04645858465651 i \\
0.08221667350938 \pm 0.03468717833984 i \\
0.08808374597041 \pm 0.02141958057287 i \\
0.09106731537025 \pm 0.00724175169196 i\end{array}$ \\
\hline
\end{tabular}



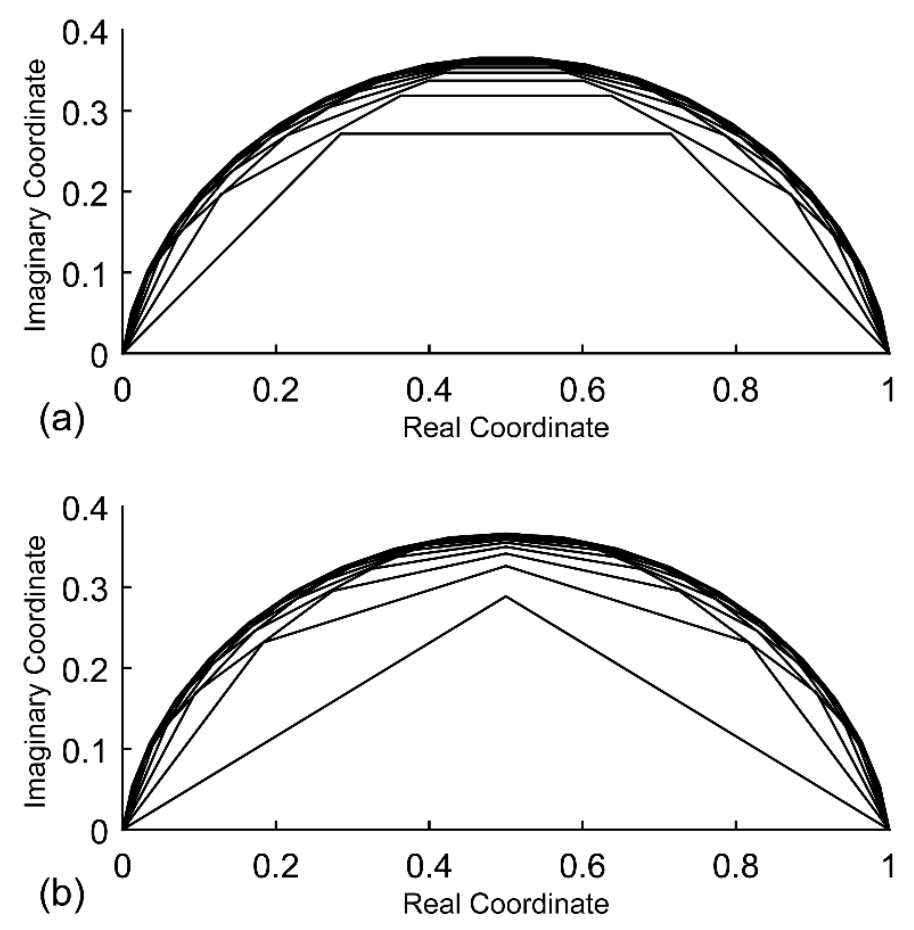

Fig. 2. Finite element meshes for (a) odd number of elements and (b) even number of elements.

It is also worth mentioning that CFEM also satisfies the following important properties:

1. CFEM is a standard finite element method, but with complex coordinate stretching. Thus, it shares similarities with perfectly matched layers (PML) used in unbounded domain modeling, which can also be viewed in terms of complex coordinate stretching [17]. The difference is that PML stretching leaves invariant one boundary point, whereas both boundary points are invariant in our method. PML finite elements lengths do not come in conjugate pairs and the one-sided DtN map is not Hermitian, consistent with PML's need to absorb energy. On the other hand, our method propagates all the information from one end of the domain to the other end without any loss of energy. This is because, although our stiffness matrix is complex symmetric, the two-sided DtN map for the entire mesh is real symmetric (Hermitian) due to complex conjugate pairs of element lengths, as shown in Appendix A.

2. Generalized eigenvalues of the stiffness matrix with respect to the mass matrix, in spite of both being complex-valued are real and non-negative. This property, which is proved in Appendix B, has implications in solving free-vibration type eigenvalue problems. It is worth noting that this phenomenon is linked to the important connection between Stieltjes rational approximants and relative rational approximants, found by Knizhnerman [18]. Specifically, he shows that there is a spectral equivalence between relative rational interpolants of the exponential and Stieltjes rational interpolants of the DtN map. As shown in [11], the latter corresponds to a spectrally matching 
three-point finite-difference scheme with symmetric negative operator, indicating that CFEM based on rational interpolants have real spectra. Rational interpolants include as a particular case the Padé approximant considered here, thus our finite-element scheme is exactly equivalent, in terms of the DtN maps, to the Padé finite-difference grid considered in [11]. Reference [11] also shows the equivalence of Padé finite-difference scheme and the spectral Galerkin method with polynomial basis functions, again in terms of the DtN maps.

3. The stiffness matrix from discretization of elliptic equations, i.e. Equation (8) with $\lambda=\alpha^{2} \geq 0$, while not Hermitian, is semi-positive stable, i.e., the real part of the eigenvalues of the stiffness matrix is equal or greater than zero. Appendix $\mathrm{C}$ contains the proof of this property.

While the discussion is limited to Laplace and Helmholtz equations, the mesh should also work for hyperbolic problems such as the wave equation. Since for CFEM, the generalized eigenvalues of the stiffness matrix with respect to the mass matrix are non-negative and real, traditional time-stepping methods would be stable. The only drawback of CFEM for hyperbolic problems is that the mass matrix is not diagonal (due to midpoint integration). However, it could be block diagonal, as the degrees of freedom are coupled only in the $x$ direction; mass lumping can be performed in the $z$ direction. Fortunately, exponential convergence of the proposed method facilitates drastic reduction in the number of elements in the $x$ direction, which translates into small block size and efficient computation.

There may be concern associated with the increased cost due to complex arithmetic. We note that this cost increase is negligible compared to the savings from significant reduction in the number of degrees of freedom facilitated by exponential convergence. Moreover, many problems involving wave propagation require complex arithmetic and complex element lengths do not increase the computational cost for these problems.

The method in the presented form is applicable to problems where the solution is needed on the edges of the subdomains. If the solutions are needed only at the corner points, if the domain geometry allows, the CFEM discretization can also be used in the transverse direction (if the material properties are pricewise constant in that direction), resulting in further reduction in the computational cost.

Since impedance-preserving property of midpoint-integrated linear finite elements extends to vector equations [7], it is expected that the proposed mesh would work equally well for electromagnetism and elasticity (similar to the development for finite difference schemes in $[5,6,12]$ ). We illustrate the applicability of the proposed approach for the special case of elastodynamics in the next section. In addition, successful application of CFEM for solving the eigenvalue problems associated with elastic layered (stratified) media can be found in [19]. 


\section{CFEM for vector equations (elastodynamics)}

We generalize CFEM for solving linear elastodynamics problems. In particular, we prove the impedance-preserving property for elastodynamics equation, and derive associated propagation factors. By observing that these propagation factors are identical to those in scalar analysis, we conclude that CFEM is applicable to vector wave equations.

\subsection{Model Problem}

Consider a two-dimensional elastic layered medium with in-plane deformation as shown in Fig. 1. Each layer is assumed to be homogeneous, but the material properties may vary between different layers. The equation representing in-plane wave propagation for the harmonic waves of the form $\overline{\mathbf{u}}(\mathbf{x}, t)=\mathbf{u}(\mathbf{x}, \omega) e^{-i \omega t}=\tilde{\mathbf{u}}(\mathbf{k}, \omega) e^{i \mathbf{k} \cdot \mathbf{x}-i \omega t}$ with no external body forces and damping, can be written as,

$$
-\nabla_{s}^{T} \boldsymbol{\sigma}-\rho \omega^{2} \mathbf{u}=\mathbf{0}
$$

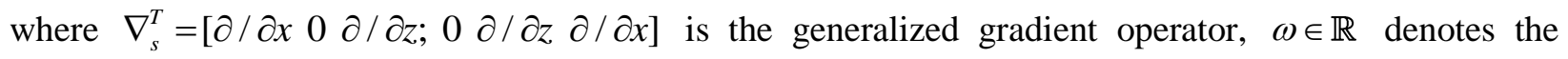
temporal frequency, $\rho$ represents the density and $\mathbf{u}=\left\{u_{x} u_{z}\right\}^{T}$ is the infinitesimal in-plane displacement vector. Appropriate Dirichlet, Neumann and/or Robin boundary conditions can be included as needed. The stress vector $\boldsymbol{\sigma}=\left\{\sigma_{x x} \sigma_{z z} \sigma_{x z}\right\}^{T}$ is related to the strain vector $\boldsymbol{\varepsilon}=\left\{\varepsilon_{x x} \varepsilon_{z z} \gamma_{x z}\right\}^{T}=\nabla_{s} \mathbf{u}$ by the stressstrain relationship:

$$
\boldsymbol{\sigma}=\left[\begin{array}{lll}
D_{11} & D_{12} & D_{13} \\
D_{12} & D_{22} & D_{23} \\
D_{13} & D_{23} & D_{33}
\end{array}\right] \boldsymbol{\varepsilon}
$$

For the special case of isotropic elasticity, material coefficients are given in terms of Lamé constants $\lambda$ and $\mu$ :

$$
D_{11}=D_{22}=(\lambda+2 \mu), D_{33}=\mu, D_{12}=\lambda, D_{13}=D_{23}=0 .
$$

Note that one can also model viscoelastic materials through frequency-dependent, complex Lamé constants. Expanding (29), we obtain,

$$
-\frac{\partial}{\partial x}\left(\mathbf{D}_{x x} \frac{\partial \mathbf{u}}{\partial x}\right)-\frac{\partial}{\partial x}\left(\mathbf{D}_{x z} \frac{\partial \mathbf{u}}{\partial z}\right)-\frac{\partial}{\partial z}\left(\mathbf{D}_{x z}^{T} \frac{\partial \mathbf{u}}{\partial z}\right)-\frac{\partial}{\partial z}\left(\mathbf{D}_{z z} \frac{\partial \mathbf{u}}{\partial z}\right)-\left(\rho \omega^{2} \mathbf{I}\right) \mathbf{u}=\mathbf{0},
$$

where $\mathbf{I}$ denotes $2 \times 2$ identity matrix and $\mathbf{D}_{x x}, \mathbf{D}_{x z}$ and $\mathbf{D}_{z z}$ are given by: 


$$
\mathbf{D}_{x x}=\left[\begin{array}{ll}
D_{11} & D_{13} \\
D_{13} & D_{33}
\end{array}\right], \quad \mathbf{D}_{x z}=\left[\begin{array}{cc}
D_{13} & D_{12} \\
D_{33} & D_{23}
\end{array}\right], \quad \mathbf{D}_{z z}=\left[\begin{array}{cc}
D_{33} & D_{23} \\
D_{23} & D_{22}
\end{array}\right] .
$$

Discretizing (32) in the $z$ direction, we obtain,

$$
\frac{\partial}{\partial x}\left(\mathbf{A}(z) \frac{\partial \mathbf{u}}{\partial x}\right)+\frac{\partial}{\partial x}\left(\mathbf{B}_{1}(z) \mathbf{u}\right)+\mathbf{B}_{2}(z) \frac{\partial \mathbf{u}}{\partial x}+\mathbf{D}(z, \omega) \mathbf{u}=\mathbf{0}
$$

where $\mathbf{A}, \mathbf{B}_{1}, \mathbf{B}_{2}$, and $\mathbf{D}$ are matrix differential operators of size $N \times N$, with $N$ being the number of degrees of freedom in the vertical direction. Equation (34) admits individual modes of the form $\mathbf{u}=\phi e^{i k_{x} x}$ where $k_{x}$ is the horizontal wavenumber. Substituting the modal form in (34) results in the dispersion relation:

$$
\left(-k_{x}^{2} \mathbf{A}+i k_{x}\left(\mathbf{B}_{1}+\mathbf{B}_{2}\right)+\mathbf{D}\right) \boldsymbol{\phi}=\mathbf{0},
$$

Considering an elastic layer from $x=0$ to $x=L$, the traction at $x=x_{0}$ takes the form,

$$
\left.\mathbf{F}\right|_{x=x_{0}}=-\left.\left(\mathbf{A} \frac{\partial \mathbf{u}}{\partial x}+\mathbf{B}_{1} \mathbf{u}\right)\right|_{x=x_{0}} \equiv-\left.\left(i k_{x} \mathbf{A}+\mathbf{B}_{1}\right) \mathbf{u}\right|_{x=x_{0}},
$$

Generalizing (36), the displacement and stress on the left and right of the layer are given by:

$$
\begin{array}{ll}
\mathbf{u}_{x=0}^{\text {exact }}=\mathbf{u}, & \mathbf{F}_{x=0}^{\text {exact }}=-\left(i k_{x} \mathbf{A}+\mathbf{B}_{1}\right) \mathbf{u}, \\
\mathbf{u}_{x=L}^{\text {exact }}=e^{i k_{x} L} \mathbf{u}, & \mathbf{F}_{x=L}^{\text {exact }}=-e^{i k_{x} L}\left(i k_{x} \mathbf{A}+\mathbf{B}_{1}\right) \mathbf{u} .
\end{array}
$$

\subsection{Impedance-preserving property and propagation factor of the mid-point integrated element}

We now focus on the segment $x=0$ to $x=x_{1}=L$. The impedance-preserving property as defined in Section 2.3 is the recovery of the exact stiffness of half-space $[0, \infty)$ after augmenting the exact stiffness of the element $[0, L]$ with the exact stiffness of right half-space $[L, \infty)$. This definition is equivalent to satisfying an equation similar to (12):

$$
\left[\begin{array}{ll}
\mathbf{K}_{11} & \mathbf{K}_{12} \\
\mathbf{K}_{21} & \mathbf{K}_{22}+\mathbf{K}_{\mathrm{HS}}
\end{array}\right]\left\{\begin{array}{l}
\mathbf{u}_{0} \\
\mathbf{u}_{L}
\end{array}\right\}=\left\{\begin{array}{c}
\mathbf{K}_{\mathrm{HS}} \mathbf{u}_{0} \\
\mathbf{0}
\end{array}\right\},
$$

where $\mathbf{u}_{0}$ and $\mathbf{u}_{L}$ are the displacements at $x=0$ and $x=L$, respectively. $\mathbf{K}_{k l}(k, l \in\{1,2\})$ correspond to the stiffness components of the layer and,

$$
\mathbf{K}_{\mathrm{HS}}=-\left(i k_{x} \mathbf{A}+\mathbf{B}_{1}\right)
$$

is the (exact) stiffness of the right half-space (see (36)). 
Drawing from the observations from the scalar problem, the impedance-preserving property is not satisfied if the layer stiffness $[0, L]$ is approximated by a regular finite element. However, like in the case of the scalar problem, it turns out that the proposed mid-point integrated layers have the impedancepreserving property, as shown in the remainder of the section.

Equation (38) can be expressed as,

$$
\left[\begin{array}{ll}
\mathbf{K}_{11}-\mathbf{K}_{\mathrm{HS}} & \mathbf{K}_{12} \\
\mathbf{K}_{21} & \mathbf{K}_{22}+\mathbf{K}_{\mathrm{HS}}
\end{array}\right]\left\{\begin{array}{l}
\mathbf{u}_{0} \\
\mathbf{u}_{L}
\end{array}\right\}=\left\{\begin{array}{l}
\mathbf{0} \\
\mathbf{0}
\end{array}\right\} .
$$

The expanded form of layer stiffness is obtained from (34) (using variational formulation followed by midpoint integration):

$$
\left[\begin{array}{ll}
\mathbf{K}_{11} & \mathbf{K}_{12} \\
\mathbf{K}_{21} & \mathbf{K}_{22}
\end{array}\right]=\frac{1}{L}\left[\begin{array}{cc}
\mathbf{A} & -\mathbf{A} \\
-\mathbf{A} & \mathbf{A}
\end{array}\right]+\frac{1}{2}\left[\begin{array}{cc}
-\mathbf{B}_{1} & -\mathbf{B}_{1} \\
\mathbf{B}_{1} & \mathbf{B}_{1}
\end{array}\right]-\frac{1}{2}\left[\begin{array}{ll}
-\mathbf{B}_{2} & \mathbf{B}_{2} \\
-\mathbf{B}_{2} & \mathbf{B}_{2}
\end{array}\right]-\frac{L}{4}\left[\begin{array}{cc}
\mathbf{D} & \mathbf{D} \\
\mathbf{D} & \mathbf{D}
\end{array}\right],
$$

Substituting (39) and (41) in (40) yields,

$$
\left(\frac{1}{L}\left[\begin{array}{cc}
\left(1+i k_{x} L\right) \mathbf{A} & -\mathbf{A} \\
-\mathbf{A} & \left(1-i k_{x} L\right) \mathbf{A}
\end{array}\right]+\frac{1}{2}\left[\begin{array}{cc}
\left(\mathbf{B}_{1}+\mathbf{B}_{2}\right) & -\left(\mathbf{B}_{1}+\mathbf{B}_{2}\right) \\
\left(\mathbf{B}_{1}+\mathbf{B}_{2}\right) & -\left(\mathbf{B}_{1}+\mathbf{B}_{2}\right)
\end{array}\right]-\frac{L}{4}\left[\begin{array}{ll}
\mathbf{D} & \mathbf{D} \\
\mathbf{D} & \mathbf{D}
\end{array}\right]\right)\left\{\begin{array}{l}
\mathbf{u}_{0} \\
\mathbf{u}_{L}
\end{array}\right\}=\left\{\begin{array}{l}
\mathbf{0} \\
\mathbf{0}
\end{array}\right\} .
$$

Since $\mathbf{u}_{0}$ and $\mathbf{u}_{L}$ satisfy the dispersion relation (35),

$$
\left(\mathbf{B}_{1}+\mathbf{B}_{2}\right) \mathbf{u}_{0}=-\left(i k_{x} \mathbf{A}+\mathbf{D} / i k_{x}\right) \mathbf{u}_{0} \quad \text { and }\left(\mathbf{B}_{1}+\mathbf{B}_{2}\right) \mathbf{u}_{L}=-\left(i k_{x} \mathbf{A}+\mathbf{D} / i k_{x}\right) \mathbf{u}_{L} .
$$

Substituting these into (42) and pre-multiplying by the nonsingular matrix $\left[L / 2-L / 2 ;-i k_{x}-i k_{x}\right]$ gives:

$$
\left[\begin{array}{ll}
\left(1+i k_{x} L / 2\right) \mathbf{A} & \left(-1+i k_{x} L / 2\right) \mathbf{A} \\
\left(1+i k_{x} L / 2\right) \mathbf{D} & \left(-1+i k_{x} L / 2\right) \mathbf{D}
\end{array}\right]\left\{\begin{array}{l}
\mathbf{u}_{0} \\
\mathbf{u}_{L}
\end{array}\right\}=\left\{\begin{array}{l}
\mathbf{0} \\
\mathbf{0}
\end{array}\right\}
$$

which is satisfied with,

$$
\mathbf{u}_{L}=P \mathbf{u}_{0}=\left(\frac{1+i k_{x} L / 2}{1-i k_{x} L / 2}\right) \mathbf{u}_{0},
$$

where $P$ is the propagation factor of the displacement using one element.

The above analysis, which is borrowed from [7], not only proves the impedance-preserving property, but also obtains the propagation factor for the displacement (45). To check the validity of CFEM for vector equations, the propagation factor is needed not just for the displacement, but for the displacementtraction pairs similar to the scalar case in (20). We now proceed to derive the propagation factor of the traction.

Given the displacement and traction at the beginning of the segment, we need to find the CFEM approximation of the displacement and stress at the end. To this end we start with the relation between the traction and displacement for the mid-point integrated element, 


$$
\left\{\begin{array}{c}
\mathbf{F}_{0} \\
-\mathbf{F}_{L}
\end{array}\right\}=\left[\begin{array}{ll}
\mathbf{K}_{11} & \mathbf{K}_{12} \\
\mathbf{K}_{21} & \mathbf{K}_{22}
\end{array}\right]\left\{\begin{array}{l}
\mathbf{u}_{0} \\
\mathbf{u}_{L}
\end{array}\right\},
$$

where the stiffness matrix of the element is given in (41). Using the impedance-preserving property (40), the above expression can be written as,

$$
\left\{\begin{array}{c}
\mathbf{F}_{0} \\
-\mathbf{F}_{L}
\end{array}\right\}=\left(\left[\begin{array}{ll}
\mathbf{K}_{11}-\mathbf{K}_{\mathrm{HS}} & \mathbf{K}_{12} \\
\mathbf{K}_{21} & \mathbf{K}_{22}+\mathbf{K}_{\mathrm{HS}}
\end{array}\right]+\left[\begin{array}{cc}
\mathbf{K}_{\mathrm{HS}} & \mathbf{0} \\
\mathbf{0} & -\mathbf{K}_{\mathrm{HS}}
\end{array}\right]\right)\left\{\begin{array}{l}
\mathbf{u}_{0} \\
\mathbf{u}_{L}
\end{array}\right\} \underset{\text { property }}{=}\left[\begin{array}{cc}
\text { impedance-preserving } \\
\mathbf{K}_{\mathrm{HS}} & \mathbf{0} \\
\mathbf{0} & -\mathbf{K}_{\mathrm{HS}}
\end{array}\right]\left\{\begin{array}{l}
\mathbf{u}_{0} \\
\mathbf{u}_{L}
\end{array}\right\},
$$

which gives the traction $\mathbf{F}_{0}$ and $\mathbf{F}_{L}$ on the left and right side of the elements:

$$
\mathbf{F}_{0}=-\left(i k_{x} \mathbf{A}+\mathbf{B}_{1}\right) \mathbf{u}_{0}, \quad \mathbf{F}_{L}=-P\left(i k_{x} \mathbf{A}+\mathbf{B}_{1}\right) \mathbf{u}_{0}=P \mathbf{F}_{0},
$$

Where (45) is used in the second equation. (45) and (48) together indicate that the propagation factors are the same for displacements and tractions. Further, comparing these with (37) indicates that the exponential propagator in (37) is approximated by the rational propagator $P$ :

$$
e^{i k_{x} L} \approx P=\left(\frac{1+i k_{x} L / 2}{1-i k_{x} L / 2}\right)
$$

The above expression is identical to the propagator for scalar equation in (25), with $\alpha=i k_{x}$. Given this identical behavior of midpoint integrated linear finite elements between scalar and vector equations, the CFEM construction for scalar equations, presented after (25), is immediately applicable for the vector wave equation.

\section{Numerical examples and discussion}

\subsection{Two-point boundary value problem: elliptic equation}

Consider a simple two-point boundary value problem on a unit interval with homogeneous Dirichlet condition on the right. Unit Neumann data is specified on the left boundary and the goal is to obtain the displacements at this boundary. The relative error is defined as,

$$
\text { error }=\frac{\left|u_{\text {exact }}-u_{\text {approx }}\right|}{\left|u_{\text {exact }}\right|+\left|u_{\text {approx }}\right|} \text {. }
$$

The analysis is performed for elliptic version of one-dimensional equation (8) with positive $\lambda=\alpha^{2}$ with $10 \leq \alpha \leq 200$. CFEM meshes with increasing number of elements (ranging from 1 through 40) are used to test the convergence of the method. Fig. 3 shows the results from the analysis for various values of $\alpha$. It can be seen that the error converges until it reaches the roundoff limit of approximately $10^{-16}$. The 
convergence is super-exponential, similar to the convergence of the optimal grids proposed in [4]. Even for a large value of $\alpha=200$, where the solution contains steeply varying functions $e^{ \pm 200}$, a coarse CFEM mesh of just 20 elements gets the relative error down to $10^{-4}$, clearly illustrating the efficiency of CFEM.

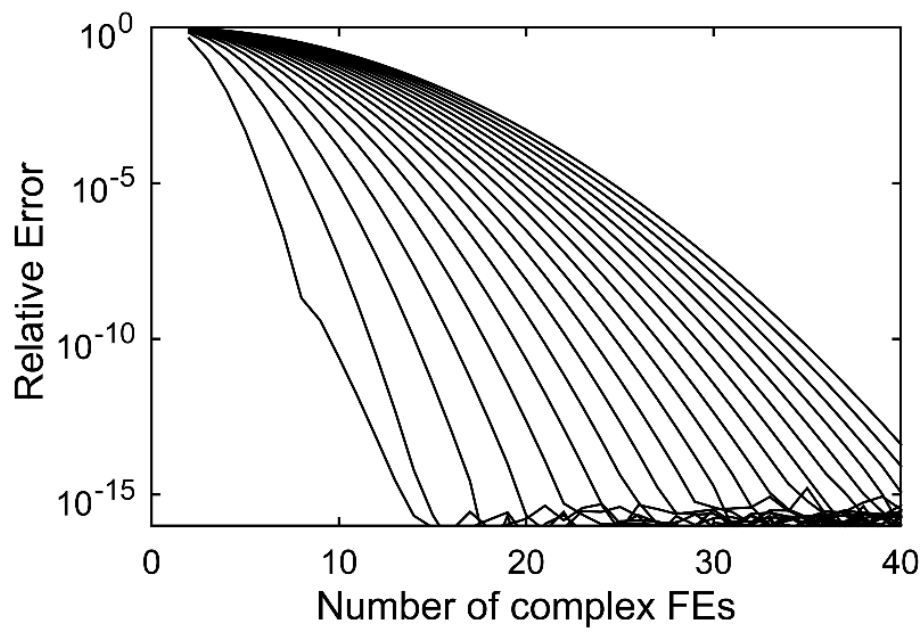

Fig. 3. Convergence for the two-point elliptic boundary value problem. The left most curve corresponds to $\alpha=10$, while the right most curve corresponds to an extremely high decay parameter of $\alpha=200$.

\subsection{Two-point boundary value problem: Helmholtz equation}

In order to assess the performance of the proposed method for wave propagation problems, we turn to the Helmholtz equation, which has a negative values of $\lambda=-\omega^{2}$, with the frequency range of $4 \leq \omega \leq 40$. As in the previous numerical experiment, homogeneous Dirichlet condition is applied on the right and unit Neumann data is specified on the left. The error as defined in (50) is plotted in Fig. 4. Superexponential convergence is again observed, but the convergence appears to occur only after the number of elements exceeds a threshold. This threshold corresponds to an average of three elements per wavelength. This is expected because, our finite element discretization is equivalent at the boundary points to Spectral Legendre-Galerkin method (see Section 3.4), and the latter has similar threshold; a more efficient approximant, e.g. Padé-Chebyshev, would make this threshold very close to the Nyquist limit of two points per wavelength $[3,4,6]$. A peculiar behavior observed in Fig. 4 is that, as the frequency is increased, the error does not converge to zero, but to a nonzero value that grows as the frequency is further increased. It turns out that reordering of elements alleviates this problem, as described below. 


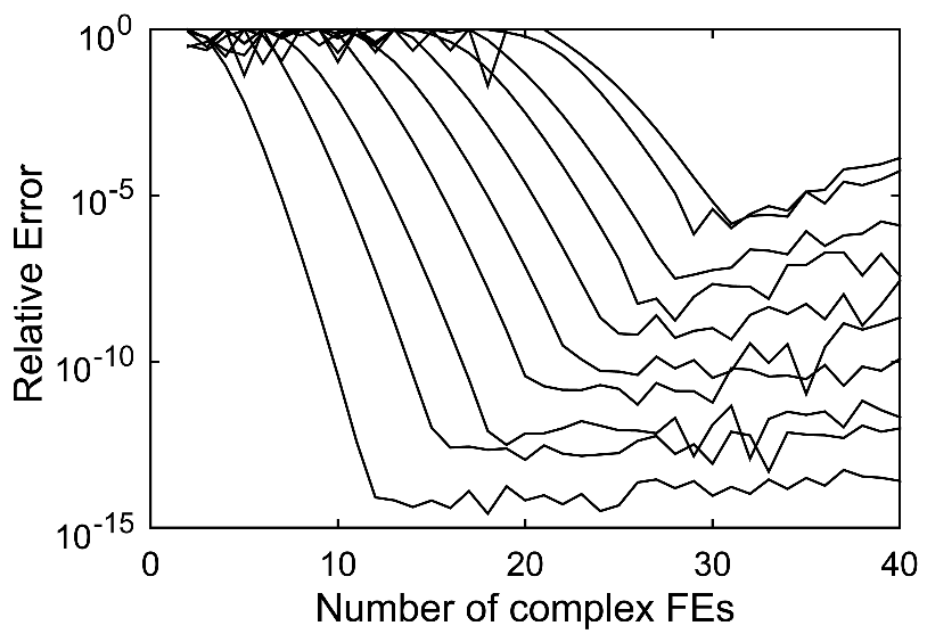

Fig. 4. Convergence for the Helmholtz boundary value problem for varying frequencies. The left most curve in each figure corresponds to the lowest frequency of $\omega=4$, while the right most curve corresponds to the highest frequency of $\omega=40$. The meshes shown in Fig. 2 are used for computation. Note that the error does not converge to a small value for high frequencies, which can be overcome by reordering of elements (cf. Fig. 6).

Element reordering: An intuitive argument for the numerical problems is as follows. Consider a wave of the form $u=a e^{ \pm i \alpha x}$ propagating through the meshes shown in Fig. 2. As we approach the center of the mesh, the imaginary part of $x$ increases, indicating that $u$ could grow exponentially. While $u$ would eventually decay back to its original order of magnitude as we approach the right edge, the growth at the center could be very large, especially for high frequencies. This translates into growth of the round-off error, resulting in significant numerical inaccuracies. An option to alleviate this problem is to pair the elements with complex conjugate lengths. Such pairing minimizes the artificial growth of the field variable and thus the round-off error. A minor disadvantage of this approach is that the symmetry of the domain geometry is not preserved after discretization. To achieve mesh symmetry, we propose to swap every alternate element in the left half of the mesh in Fig. 2, with its symmetric counterparts on the right (few examples of rearranged meshes are given in Fig. 5). This enforces alternating signs for imaginary parts of element lengths, thus reducing the growth of the imaginary part of the coordinate $x$. With the new element ordering, the solution to the Helmholtz equation improved considerably, as shown in Fig. 6.

Note that reordering would accordingly affect the condition number of the dynamic stiffness matrix of the discretized Helmholtz problem in Equation (8) with $\lambda=-\omega^{2}$, i.e. $\mathcal{K}_{d}=\mathcal{K}-\omega^{2} \mathcal{M}$ (unlike the generalized eigenvalues that are invariant to the element ordering, as shown in Appendix B). As an example, Fig. 7 (a) shows the condition number of the dynamic stiffness matrix for the Helmholtz problem with $\omega=40$ normalized by the condition number of the discretized system using spectral finite element method (SFEM) with the same number of nodes and thus the same level of accuracy (we later 
show that CFEM and SFEM give same accuracy for a total same number of nodes in the mesh - see Fig. 8). Similarly, Fig. 7 (b) shows the normalized condition number after element reordering. Clearly, before element reordering, CFEM is conditioned much worse than SFEM, with the normalized condition number growing exponentially with the number of CFEM elements. After reordering though, the normalized condition number is less than 1, indicating that CFEM with reordering is slightly better conditioned than SFEM, confirming that it is best to use CFEM with element reordering.
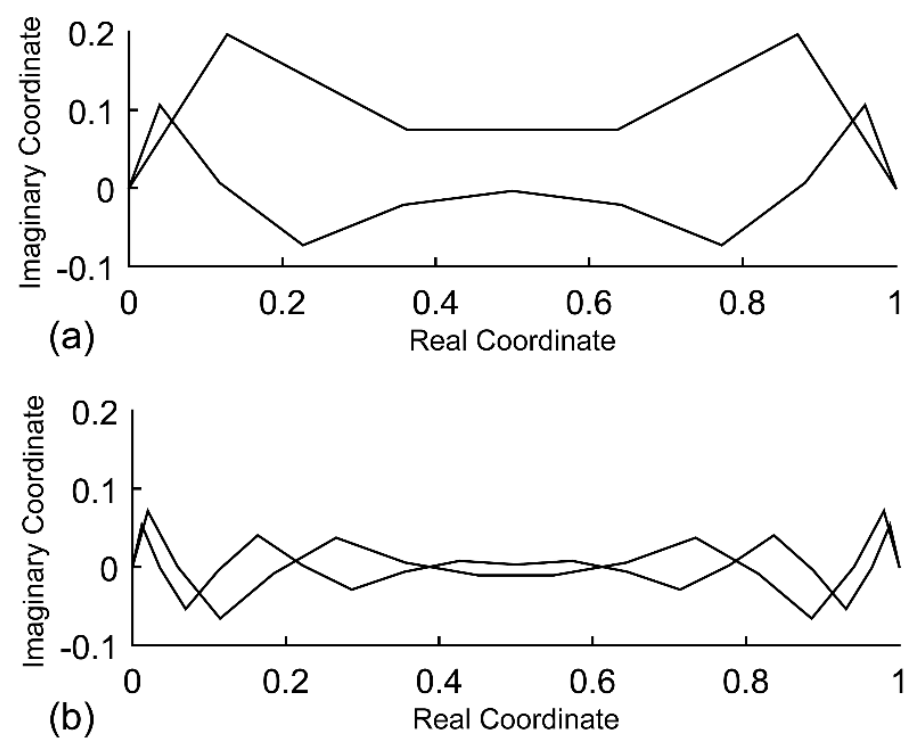

Fig. 5. Representative meshes after element reordering for (a) 5-element and 10-element meshes and (b) 15 -element and 20-element meshes. Note that the bounds of imaginary part of the nodal coordinates are reducing with refinement. This counters the numerical growth and helps achieve better convergence (compare these meshes with the meshes in Fig. 2).

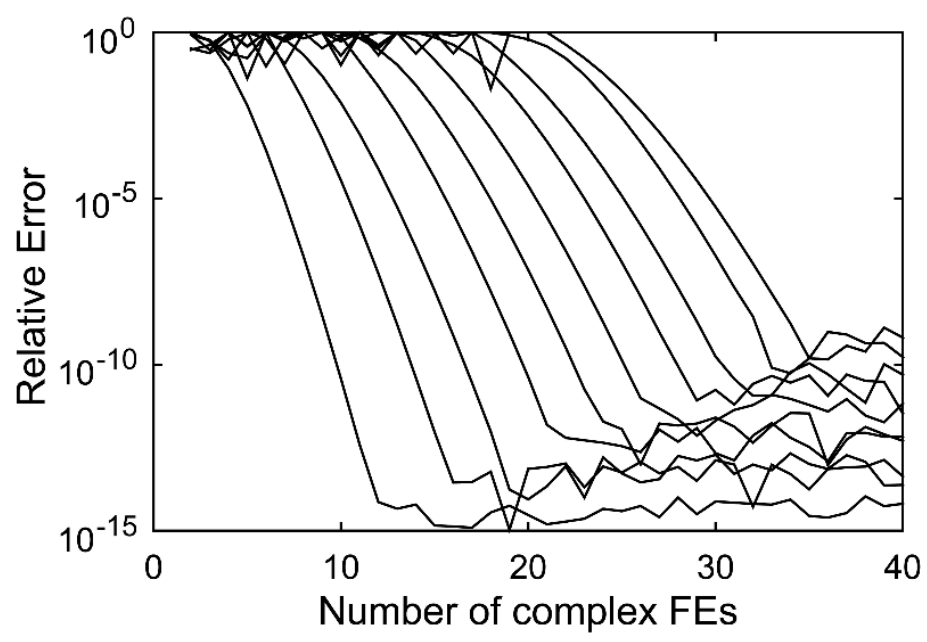

Fig. 6. Convergence for the two-point Helmholtz boundary value problem after element reordering shown in Fig. 5. In comparison with Fig. 4, the error is converging to a much smaller value, illustrating the 
effectiveness of element reordering.

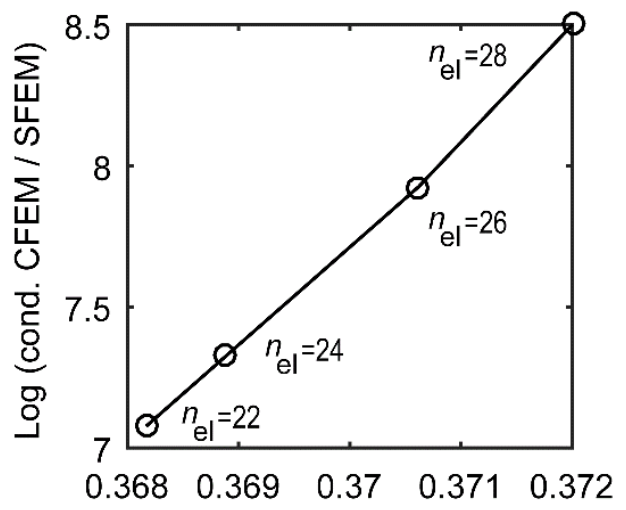

(a) $\max$ (imaginary coordinate)

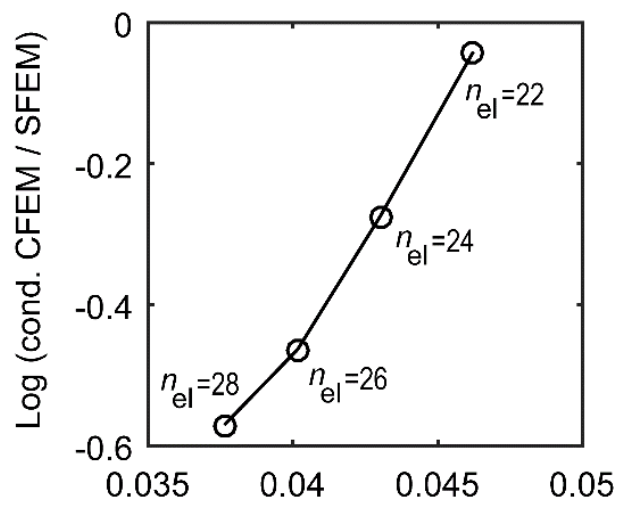

(b)

Fig. 7. Condition numbers of CFEM (normalized with respect to spectral FEM) for the Helmholtz problem with $\omega=40$, (a) without element reordering and (b) with element reordering.

A note on numerical dispersion: A common issue encountered in finite element solution of Helmholtz problem is numerical dispersion, where the waveform is approximated with inexact wavelength resulting in accumulating phase errors for large domains [20]. In contrast with traditional FEM, CFEM results in exponentially convergent solutions directly at select points in the domain, and there is no approximation of the waveform over the domain and associated accumulation of phase error. Thus, the issue of dispersion error does not exist in the context of CFEM.

\subsection{Comparison with spectral FEM}

In order to investigate the efficiency of the CFEM, we also compare the convergence results of CFEM with spectral finite elements (SFEM) using Lobatto polynomials as discussed in [21]. Results are reported in Fig. 8 for the lowest and highest values of $\alpha$ and $\omega$ for the elliptic and Helmholtz two-point boundary value problems, respectively. Fig. 8 (a) shows that, for the elliptic equation, performance of CFEM is slightly better than SFEM for $\alpha=10$ and the disparity between the two methods becomes larger with increasing $\alpha$. However as shown in Fig. 8 (b) for the Helmholtz equation which has a harmonic response, both methods have the same convergence for different frequencies, indicating that both CFEM and SFEM would require the same number of degrees of freedom. CFEM is however significantly more efficient than SFEM since CFEM matrices are sparse (tridiagonal in this case), compared to full SFEM matrices. An additional advantage of CFEM is that it can easily be implemented into existing finite element codes with simple change of element lengths and integration rule. 


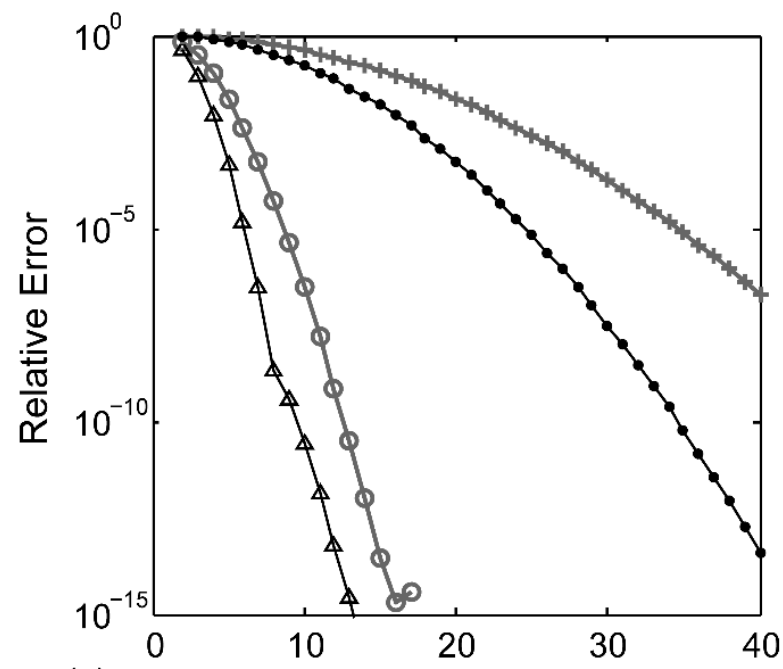

(a)

dof

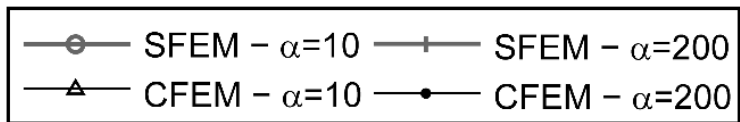

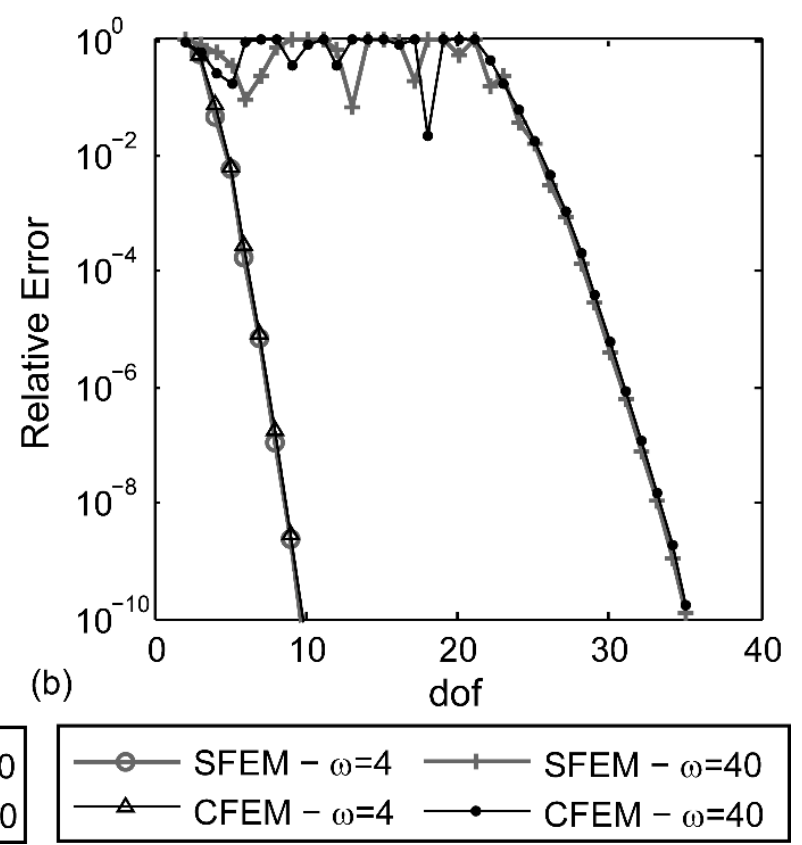

Fig. 8. Convergence curves for (a) the two-point elliptic boundary value problem and (b) the two-point Helmholtz boundary value problem using CFEM and spectral FEM.

\subsection{Two-dimensional layer: Laplace equation}

Extending the one-dimensional examples to two-dimensional setting, we consider a long layer of size $1 \times 10$, governed by Laplace equation with unit coefficients. Homogeneous Dirichlet boundary condition is applied at the bottom and homogeneous Neumann condition is applied on the top and right edges. In order to focus on the effect of $x$ discretization, we use a fine mesh of 200 elements in the $z$ direction. The excitation (specified Neumann data) at the left boundary is given by the analytic function,

$$
-G \frac{\partial u}{\partial x}=e^{16+4 / y /(\mathrm{y}-1)},
$$

which excites various vertical harmonics, resulting in wide-ranging decay rates in the horizontal direction. The reference solution is taken as the converged FEM solution. The response on the left edge is computed and the resulting error $e_{r}=\left\|\mathbf{u}-\mathbf{u}^{\text {ref }}\right\|_{2} /\left\|\mathbf{u}^{\text {ref }}\right\|_{2}$ is examined, where $\mathbf{u}$ and $\mathbf{u}^{\text {ref }}$ are the calculated and reference displacement vectors, respectively.

Convergence with mesh refinement for CFEM is shown in Fig. 9 (a). Convergence analysis is also performed for regular FEM and the results are shown in Fig. 9 (b). Clearly CFEM shows exponential convergence, while regular FEM shows expected second-order convergence (note that the left plot is in semi-log scale, while the right plot is in log-log scale). Quantitatively, just 10 CFEM elements are 
sufficient to achieve an error of less than 1\%, whereas uniform FE mesh requires more than 100 elements for the same accuracy. When the desired error is reduced $0.01 \%$, we require just 14 CFEM elements, as opposed to approximately 1000 regular FE.
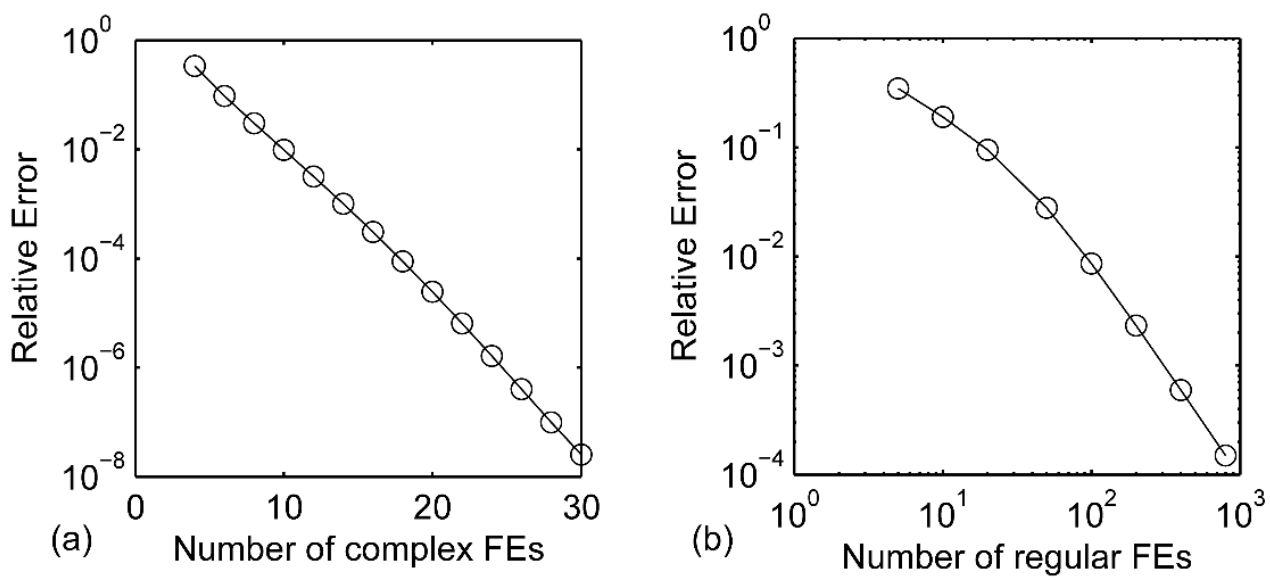

Fig. 9. Convergence of CFEM for Laplace equation in 2D. Note the exponential convergence in CFEM discretization as opposed to algebraic convergence of regular FEM (left plot is semi-log and the right plot is $\log -\log$ ). For $1 \%$ relative error, one would need 10 CFEM elements as opposed to 100 regular elements.

$0.01 \%$ relative error requires just 14 CFEM elements, as opposed to 1000 regular finite elements.

\subsection{Two-dimensional layer: Helmholtz equation}

The 2D problem in the previous section is solved now for Helmholtz equation with frequency of $\omega=3$. The frequency is chosen to ensure existence of significant evanescent as well as propagating waves. Since the domain is bounded, in order to eliminate complications due to resonance, we used $G=1+0.01 i$ in the governing equation (1) to damp out the resonances. Convergence analysis similar to that performed for the Laplace equation is performed for this problem. The results are shown in Fig. 10 (a) for CFEM and Fig. 10 (b) for regular FEM. The plot also contains the convergence results for the field variable at $x=10$. Possibly because of resonances and oscillations, the CFEM convergence is slowed compared to the Laplace equation. However, it is much faster compared to the second order convergence of regular FEM. Quantitatively, a desired error of $1 \%$ requires just 17 CFEM elements, as opposed to 400 regular finite elements. When the desired error is $0.1 \%$, the disparity increases: 20 elements for CFEM as opposed to more than 1000 elements for regular FEM. Note that, due to exponential convergence, CFEM does not suffer from adverse consequences of pollution effects of the dispersion error associated with regular FEM [22]. 

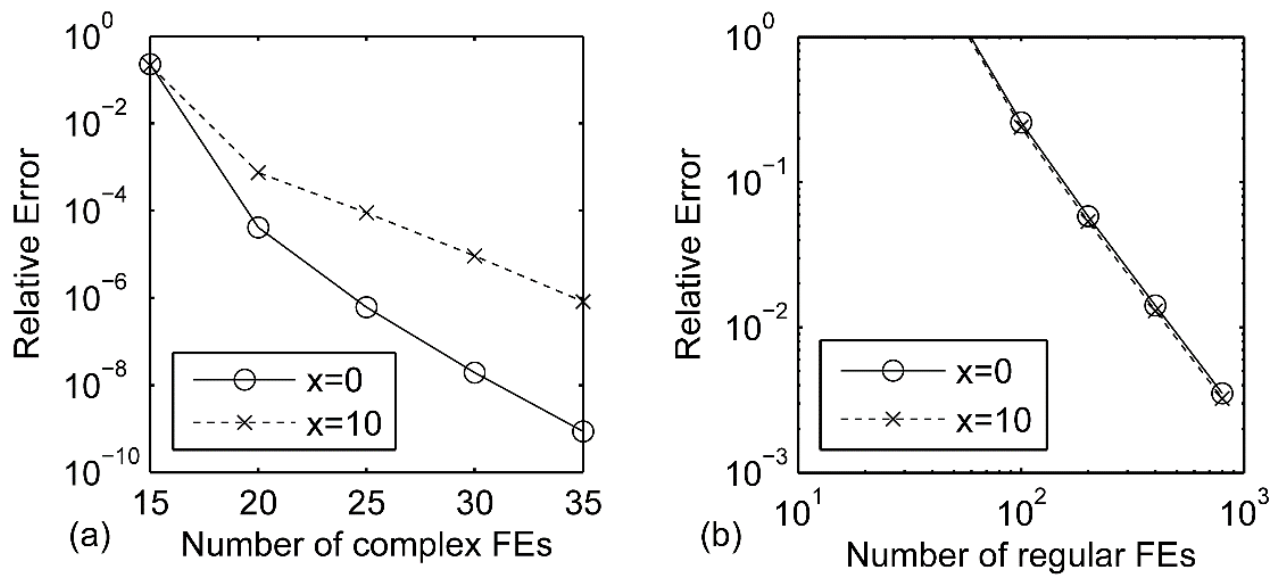

Fig. 10. Convergence of CFEM for Helmholtz equation in a two-dimensional setting. Note the exponential convergence in CFEM. For $1 \%$ relative error, 17 CFEM elements are needed as opposed to 400 regular finite elements. $0.1 \%$ relative error requires just $20 \mathrm{CFEM}$ elements, as opposed to more than 1000 regular finite elements.

\subsection{Two-dimensional Helmholtz problem with multiple subdomains}

To illustrate the applicability of the proposed method for problems with multiple subdomains, the modulus of the right half $(5<x<10)$ is modified to $G=2+0.02 i$. The subdomains $(0,5)$ and $(5,10)$ are discretized separately using CFEM, with nodes shared at $x=5$. Accuracy is expected at $x=0,5,10$, while the remaining nodes have complex coordinates. The convergence of the solution at these locations is plotted in Fig. 11 (a). Similar to the previous example, the convergence is worse compared to the Laplace equation, but much faster than that for regular finite element discretization, which is shown in Fig. 11 (b).

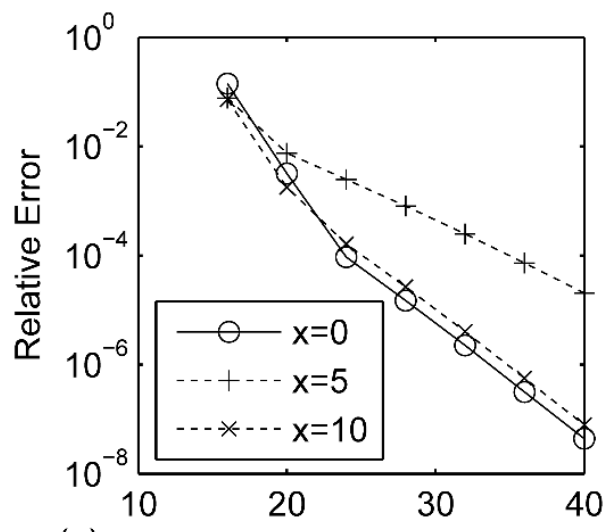

(a) Number of complex FEs

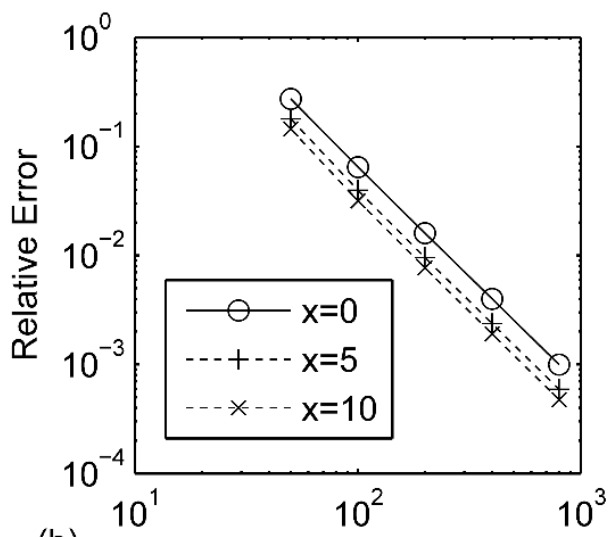

(b) Number of regular FEs

Fig. 11. Convergence for multiple-subdomain problem. Note the exponential convergence of CFEM. For $1 \%$ relative error, we would need 20 CFEM elements as opposed to 300 regular finite elements. $0.1 \%$ error tolerance requires $28 \mathrm{CFEM}$ elements as opposed to more than 800 regular finite elements. 


\subsection{Two-dimensional elastodynamics problem with multiple subdomains}

The 2D multi-subdomain problem of the previous section is now solved for time-harmonic elastodynamics equation given in (29) with frequency $\omega=3$. The shear modulus of the left half and right half are chosen as $G=1+0.01 i$ and $G=2+0.02 i$, respectively. The Poisson's ratio is taken as $v=0.35$, the density is assumed to be $\rho=1$ and the following horizontal traction is applied on the left boundary of the domain at $x=0$ :

$$
\left.\left\{\begin{array}{c}
\sigma_{x x} \\
\sigma_{x y}
\end{array}\right\}\right|_{x=0}=-\left.\left(\mathbf{G}_{x x} \frac{\partial}{\partial x}+\mathbf{G}_{x y} \frac{\partial}{\partial y}\right) \mathbf{u}\right|_{x=0}=\left\{\begin{array}{c}
e^{16+4 / y(y-1)} \\
0
\end{array}\right\}
$$

Similar to the previous example the convergence of the displacements at the interfaces $x=5,10,15$ are shown in Fig. 12 using CFEM and regular FEM with the relative error including both horizontal and vertical components of the displacement. It can be observed that, like in the previous example, CFEM requires much fewer elements compared to regular FEM.
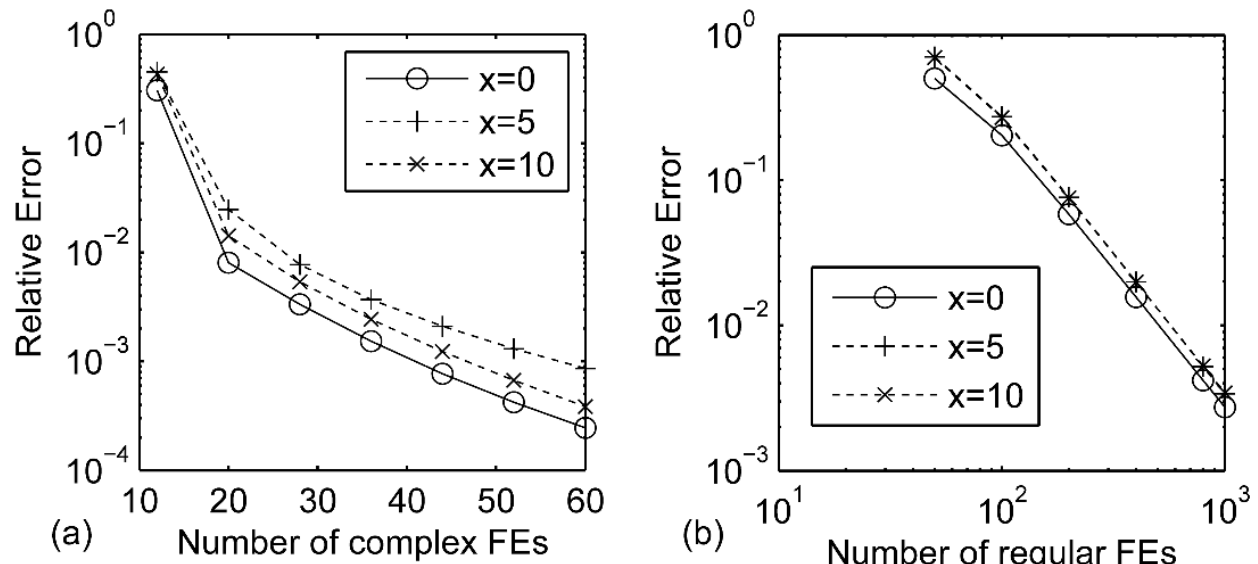

Fig. 12. Convergence for multiple-subdomain elastodynamics problem. For $1 \%$ relative error, we would need 28 CFEM elements as opposed to 500 regular finite elements. $0.1 \%$ error tolerance requires 60

CFEM elements as opposed to more than 1000 regular finite elements.

\section{Conclusions}

We introduced a novel modification of linear finite element discretization to achieve exponential convergence of the solution at select points in the domain. The main idea is to obtain exponentially convergent approximation for the DtN map of the sub-domains spanning between the points of interest. By employing the midpoint integration rule and an unconventional mapping of the finite element mesh into the complex space, we are able to achieve high accuracy at the edges of the sub-domain with a very coarse discretization of the interior. The development is facilitated by linking midpoint-integrated linear finite element discretization to Crank-Nicolson stepping of the associated first order form, which in turn is 
related to Padé approximants of the exponential function. The parameters of the Padé approximants are translated back to the parameters of finite element discretization (element lengths). The resulting element lengths are complex-valued, and the method is named Complex-length Finite Element Method (CFEM). CFEM inherits the exponential convergence from the underlying Padé approximants. Exponential convergence is verified with a variety of numerical experiments involving Laplace, Helmholtz and elastodynamics equations. The examples also indicate that, for practical error tolerance, CFEM facilitates reduction of the number of elements by an order of magnitude resulting in significant savings in computational cost.

The current paper indicates promise of CFEM, but further investigations and enhancements are necessary, especially because of the unconventional nature of the method. These investigations include: (a) deeper mathematical understanding of the underlying approximation properties; (b) further investigation of numerical properties of the resulting discretizations; (c) extension to tensor product CFEM discretizations for higher dimensions including resolution of possible stability issues associated with midpoint integration in higher dimensions; (d) extension of the method for hyperbolic problems; and (e) possible extension to topologically triangular meshes thus to unstructured meshes.

\section{Acknowledgements}

The authors wish to thank Leonid Knizhnerman for useful discussions. The work is partially supported by National Science Foundation (Grant No. DMS-1016514) and Schlumberger Technology Corporation.

\section{Appendix A: Proof that DtN map is Hermitian}

Consider the following equation similar to (8) with $\lambda=\alpha^{2} \in \mathbb{R}$,

$$
-\frac{\partial^{2} u}{\partial x^{2}}+\alpha^{2} u=0, \text { for } 0<x<L
$$

From (17), the stiffness matrix of any mid-point integrated element $\left(\mathcal{K}_{\text {elem }}+\alpha^{2} \mathcal{M}_{\text {elem }}\right)$, is of the form $\mathcal{K}_{d}^{j}=\left[\begin{array}{llll}a & b ; & b & a\end{array}\right]$, where $a=1 / L_{j}+\alpha^{2} L_{j} / 4$ and $b=-1 / L_{j}+\alpha^{2} L_{j} / 4$. The assembled stiffness matrix for a pair of elements with complex conjugate lengths $L$ and $\bar{L}$ takes the form, 


$$
\left(\mathcal{K}_{L, \bar{L}}+\alpha^{2} \mathcal{M}_{L, \bar{L}}\right)=\left[\begin{array}{ccc}
a & b & \\
b & a+\bar{a} & \bar{b} \\
& \bar{b} & \bar{a}
\end{array}\right]
$$

Condensing out the middle node and using the impedance preserving property (14), i.e., $a^{2}-b^{2}=\alpha^{2}$, the $2 \times 2$ DtN map of (A2) takes the form,

$$
\mathbf{S}_{L, \bar{L}}=\frac{1}{2 \mathfrak{R}(a)}\left[\begin{array}{cc}
\alpha^{2}+|a|^{2} & -|b|^{2} \\
-|b|^{2} & \alpha^{2}+|a|^{2}
\end{array}\right] .
$$

Equation (A3) indicates that $\mathbf{S}_{L, \bar{L}}$ is a real symmetric (Hermitian) matrix similar to the stiffness matrix of an element with real length. Since the CFEM element lengths are either real or complex conjugate pairs, the assembly of DtN maps for all elements and consequently the DtN map of the entire mesh will be real symmetric (Hermitian). This is true not only for positive $\alpha^{2}$ (elliptic equation) but also for negative $\alpha^{2}$ (Helmholtz equation).

\section{Appendix B: Proof that eigenvalues of $(\mathcal{K}, \mathcal{M})$ are real and nonnegative}

Discretizing the weak form of (A1) leads to the system of equations $\left(\mathcal{K}+\alpha^{2} \mathcal{M}\right)$. Generalized eigenvalues of the pencil $(\mathcal{K}, \mathcal{M})$ are real and non-negative, if and only if all values of $\alpha$ with singular $\left(\mathcal{K}+\alpha^{2} \mathcal{M}\right)$ are purely imaginary. We prove this using the following lemmas.

Lemma B1. For a mesh with elements $L_{1}, \ldots, L_{n}$, the propagator of the entire mesh $P_{L_{1}, \ldots, L_{n}}(\alpha)= \pm 1$ for any $\alpha$ with singular $\left(\mathcal{K}+\alpha^{2} \mathcal{M}\right)$.

Proof. The two-point DtN of the entire mesh takes the form,

$$
\mathbf{S}=\left[\begin{array}{cc}
S_{\text {diag }} & S_{\text {off }} \\
S_{\text {off }} & S_{\text {diag }}
\end{array}\right]
$$

The diagonal entries of DtN map are the same due to the mirror symmetry of the mesh (because $\mathbf{S}$ is invariant of element ordering as discussed in Section 3.3 and we simply choose the mesh that is mirror symmetric to make the claim). Similar to (12), the impedance-preserving property of the DtN map can be written as, 


$$
\left[\begin{array}{cc}
S_{\text {diag }}-K_{\text {halfspace }} & S_{\text {off }} \\
S_{\text {off }} & S_{\text {diag }}+K_{\text {halfspace }}
\end{array}\right]\left\{\begin{array}{l}
u_{0} \\
u_{1}
\end{array}\right\}=\left\{\begin{array}{l}
0 \\
0
\end{array}\right\} .
$$

Given that $K_{\text {halspace }}=\alpha$ and using the propagator for $u_{1}=P_{L_{1}, \ldots, L_{n}} u_{0},(\mathrm{~B} 2)$ becomes,

$$
\left[\begin{array}{cc}
S_{\text {diag }}-\alpha & S_{\text {off }} \\
S_{\text {off }} & S_{\text {diag }}+\alpha
\end{array}\right]\left\{\begin{array}{c}
1 \\
P_{L_{1}, \ldots, L_{n}}
\end{array}\right\}=\left\{\begin{array}{l}
0 \\
0
\end{array}\right\} .
$$

Using (B3) and the impedance-preserving property, $S_{\text {diag }}^{2}-S_{\text {off }}^{2}=\alpha^{2}$ (see (14)), we obtain,

$$
P_{L_{1}, \ldots, L_{n}}= \pm \sqrt{\frac{1-\alpha / S_{\mathrm{diag}}}{1+\alpha / S_{\mathrm{diag}}}}
$$

Singularity of $\left(\mathcal{K}+\alpha^{2} \mathcal{M}\right)$ implies the singularity (rank-deficiency) of the Schur complement $\mathbf{S}$. However due to impedance-preserving property, $\operatorname{det}(\mathbf{S})=S_{\text {diag }}^{2}-S_{\text {off }}^{2}=\alpha^{2}$, which is finite. This can only occur if the components of the DtN map, $S_{\text {diag }}, S_{\text {off }} \rightarrow \infty$. With this, (B4) becomes,

$$
P_{L_{1}, \ldots, L_{n}}(\alpha)= \pm 1
$$

which proves the assertion. An alternative proof can be obtained by utilizing the isomorphism between the propagators obtained via CFEM and the two-sided optimal grid in Section 3.3 of [23], but is not presented here.

Lemma B2. For $\alpha=\alpha_{R}+i \alpha_{I}$ with nonzero $\alpha_{R}$, the absolute value of the propagator cannot be unity $\left|P_{L_{1}, \ldots, L_{n}}(\alpha)\right| \neq 1$.

Proof. Following (26) the propagator for the entire mesh with elements $L_{1}, \ldots, L_{n}$ can be written as the multiplication of the propagators of individual elements, i.e.,

$$
P_{L_{1}, \ldots, L_{n}}(\alpha)=P_{L_{n}} P_{L_{n-1}} \cdots P_{L_{2}} P_{L_{1}}
$$

We start with the propagator for a pair of CFEM elements with complex conjugate lengths $L$ and $\bar{L}$ (obtained using (26)): 


$$
P_{L, \bar{L}}(\alpha)=\left(\frac{1+\alpha L / 2}{1-\alpha L / 2}\right)\left(\frac{1+\alpha \bar{L} / 2}{1-\alpha \bar{L} / 2}\right)
$$

Considering $L=L_{R}+i L_{I}$, the propagator can be written as,

$$
P_{L, \bar{L}}(\alpha)=\frac{\left(\mathcal{X}+L_{R} \alpha_{R}\right)+i\left(\mathcal{Y}+L_{R} \alpha_{I}\right)}{\left(\mathcal{X}-L_{R} \alpha_{R}\right)+i\left(\mathcal{Y}-L_{R} \alpha_{I}\right)}
$$

where $\mathcal{X}=\left(\alpha_{R}^{2}-\alpha_{I}^{2}\right)|L|^{2} / 4+1$ and $\mathcal{Y}=\alpha_{R} \alpha_{I}|L|^{2} / 2$. The absolute value takes the form,

$$
\left|P_{L, \bar{L}}(\alpha)\right|=\sqrt{\frac{\mathcal{Q}+\mathcal{E}}{\mathcal{Q}}}
$$

where $\mathcal{Q}=\left(\mathcal{X}-L_{R} \alpha_{R}\right)^{2}+\left(\mathcal{Y}-L_{R} \alpha_{I}\right)^{2}$ and $\mathcal{E}=4 L_{R}\left(\mathcal{X} \alpha_{R}+\mathcal{Y} \alpha_{I}\right)=\alpha_{R} L_{R}\left(|L|^{2}|\alpha|^{2}+4\right)$. It can be seen that $\left|P_{L, \bar{L}}(\alpha)\right|>1$ for $\alpha_{R}>0$ and $\left|P_{L, \bar{L}}(\alpha)\right|<1$ for $\alpha_{R}<0$.

For odd number of CFEM elements, in addition to the complex conjugate lengths, there is a single element with real length $L_{R}$. It immediately follows from (25) that $\left|P_{L_{R}}(\alpha)\right|>1$ for $\alpha_{R}>0$, and $\left|P_{L_{R}}(\alpha)\right|<1$ for $\alpha_{R}<0$.

Combining the above observations into (B6), we note that $\left|P_{L_{1}, \ldots, L_{n}}(\alpha)\right| \neq 1$ for nonzero $\alpha_{R}$ :

$$
\left\{\begin{array}{l}
\left|P_{L_{i}, \bar{L}_{i}}\right|,\left|P_{L_{R}}\right|>1 \quad(i=1, \ldots, m) \Rightarrow\left|P_{L_{1}, \ldots, L_{n}}\right|=\left|P_{L_{R}}\right| \times \prod_{i=1}^{m}\left|P_{L_{i}, \bar{L}_{i}}\right|>1, \quad \text { for } \quad \alpha_{R}>0, \\
\left|P_{L_{i}, \bar{L}_{i}}\right|,\left|P_{L_{R}}\right|<1 \quad(i=1, \ldots, m) \Rightarrow\left|P_{L_{1}, \ldots, L_{n}}\right|=\left|P_{L_{R}}\right| \times \prod_{i=1}^{m}\left|P_{L_{i}, \bar{L}_{i}}\right|<1, \quad \text { for } \quad \alpha_{R}<0,
\end{array}\right.
$$

which concludes the proof.

Proof of the main claim: Lemma B1 states that singularity of singular $\left(\mathcal{K}+\alpha^{2} \mathcal{M}\right)$ implies $P_{L_{1}, \ldots, L_{n}}(\alpha)= \pm 1$. On the other hand, from Lemma B2, any $\alpha$ that is not purely imaginary would result in $P_{L_{1}, \ldots, L_{n}}(\alpha) \neq \pm 1$, which indicates $P_{L_{1}, \ldots, L_{n}}(\alpha)= \pm 1$ is possible only for purely imaginary $\alpha$. Thus, singularity of $\left(\mathcal{K}+\alpha^{2} \mathcal{M}\right)$ implies that $\alpha$ must be purely imaginary, which in turn implies that the generalized eigenvalues of $\mathcal{K}$ with respect to $\mathcal{M}$ are real and nonnegative. 
Remark: Note that, in spite of real spectrum, the pencil $(\mathcal{K}, \mathcal{M})$ is not Hermitian. Its left eigenvectors are complex, except at the boundary where they are real. Further, the left and right eigenvectors are equal to each other without conjugation, so the spectral measure is generally complex (except its restriction at the boundary where it is real positive).

\section{Appendix C: Proof of semi-positive stability of $\mathcal{K}+\alpha^{2} \mathcal{M}$ for $\alpha^{2} \geq 0$}

To show that the stiffness matrix $\mathcal{K}_{d}^{N \times N}=\mathcal{K}+\alpha^{2} \mathcal{M}$ is semi-positive stable, i.e. the real part of the eigenvalues are nonnegative $\left(\Re\left(\mu_{i}\right) \geq 0, i=1, \ldots, N\right)$, we use the following Lemma and then observe that the Hermitian part of the stiffness matrix is indeed semi-positive definite.

Lemma C1. Stiffness matrix $\mathcal{K}^{d}$ is semi-positive stable if its Hermitian part $\mathcal{H}\left(\mathcal{K}^{d}\right)$ is semi-positive definite.

Proof. Let $\mu_{i}$ and $\boldsymbol{v}_{i}$ be an eigenvalue and corresponding eigenvector of $\mathcal{K}^{d}$. Then, $\mathcal{K}^{d} \boldsymbol{v}_{i}=\mu_{i} \mathbf{v}_{i}$ and $\mathbf{v}_{i}^{\dagger} \mathcal{K}_{d}^{\dagger}=\bar{\mu}_{i} \mathbf{v}_{i}^{\dagger}$, where $\dagger$ denotes the conjugate transpose. Pre- and post-multiplying these expressions by $\boldsymbol{v}_{i}^{\dagger}$ and $\boldsymbol{v}_{i}$ respectively and averaging them results in,

$$
\mathfrak{R}\left(\mu_{i}\right)=\frac{1}{2}\left(\mu_{i}+\bar{\mu}_{i}\right)=\boldsymbol{v}_{i}^{\dagger} \frac{1}{2}\left(\mathcal{K}_{d}+\mathcal{K}_{d}^{\dagger}\right) \boldsymbol{v}_{i} / \mathbf{v}_{i}^{\dagger} \boldsymbol{v}_{i}=\mathbf{v}_{i}^{\dagger} \mathcal{H}\left(\mathcal{K}^{d}\right) \boldsymbol{v}_{i} / \mathbf{v}_{i}^{\dagger} \mathbf{v}_{i} \geq 0, \quad i=1, \ldots, N
$$

Semi-positive definiteness of $\mathcal{H}\left(\mathcal{K}_{d}\right)$ follows from semi-positive definiteness of Hermitian part of element matrices, which can be written as (from (17) with $\lambda=\alpha^{2}$ ),

$$
\mathcal{H}\left(\mathcal{K}_{d}^{\text {elem }}\right)=\frac{\Re\left(L_{j}\right)}{\left|L_{j}\right|^{2}}\left[\begin{array}{cc}
1 & -1 \\
-1 & 1
\end{array}\right]+\alpha^{2} \frac{\Re\left(L_{j}\right)}{4}\left[\begin{array}{ll}
1 & 1 \\
1 & 1
\end{array}\right]=\tilde{\mathcal{K}}_{\text {elem }}+\alpha^{2} \tilde{\mathcal{M}}_{\text {elem }} .
$$

Proof of the main claim: Clearly $\tilde{\mathcal{K}}_{\text {elem }}$ and $\tilde{\mathcal{M}}_{\text {elem }}$ are semi-positive definite and, for $\alpha^{2} \geq 0$, $\mathcal{H}\left(\mathcal{K}_{d}^{\text {elem }}\right)$ is semi-positive definite. Consequently the Hermitian part for the assembled stiffness matrix $\mathcal{H}\left(\mathcal{K}_{d}\right)$ is semi-positive definite. This, combined with Lemma $\mathrm{C} 1$, proves the assertion that $\mathcal{K}+\alpha^{2} \mathcal{M}$ is semi-positive stable for $\alpha^{2} \geq 0$.

\section{References}

[1] B. Fornberg, A practical guide to pseudospectral methods., Cambridge University Press, U.K., 1996.

[2] L.N. Trefethen, Spectral methods in MATLAB, SIAM, Philadelphia, PA, 2000.

[3] V. Druskin, L. Knizhnerman, Gaussian spectral rules for the three-point second differences: I. A twopoint positive definite problem in a semi-infinite domain, SIAM J. Numer. Anal. 37 (2000) 403-422. 
[4] V. Druskin, L. Knizhnerman, Gaussian spectral rules for second order finite-difference schemes, Numer. Algorithms. 25 (2000) 139-159.

[5] S. Asvadurov, V. Druskin, L. Knizhnerman, Application of the difference Gaussian rules to solution of hyperbolic problems, J. Comput. Phys. 158 (2000) 116-135.

[6] S. Asvadurov, V. Druskin, L. Knizhnerman, Application of the difference Gaussian rules to solution of hyperbolic problems II. Global expansion, J. Comput. Phys. 175 (2002) 24-49.

[7] M.N. Guddati, Arbitrarily wide-angle wave equations for complex media, Comput. Meth. Appl. Mech. Eng. 195 (2006) 65-93.

[8] M.N. Guddati, K.W. Lim, Continued fraction absorbing boundary conditions for convex polygonal domains, Int. J. Numer. Methods Eng. 66 (2006) 949-977.

[9] M.A. Zahid, M.N. Guddati, Padded continued fraction absorbing boundary conditions for dispersive waves, Comput. Meth. Appl. Mech. Eng. 195 (2006) 3797-3819.

[10] D. Ingerman, V. Druskin, L. Knizhnerman, Optimal finite difference grids and rational approximations of the square root I. Elliptic problems, Commun. Pure Appl. Math. 53 (2000) 10391066.

[11] V. Druskin, S. Moskow, Three-point finite-difference schemes, Pade and the spectral Galerkin method. I. One-sided impedance approximation, Math. Comput. 71 (2002) 995-1019.

[12] S. Davydycheva, V. Druskin, T. Habashy, An efficient finite-difference scheme for electromagnetic logging in 3D anisotropic inhomogeneous media, Geophysics. 68 (2003) 1525-1536.

[13] G. Nemeth, Relative rational approximation of function exp(x), Math. Notes. 21 (1977) 325-328.

[14] G.A. Baker, P.R. Graves-Morris, Padé approximants., 2nd ed., Cambridge University Press, New York, NY, 1996.

[15] A.A. Gonchar, E.A. Rakhmanov, Equilibrium distributions and degree of rational approximation of analytic-functions, Math. USSR Sb. 134 (1987) 305-348.

[16] E.B. Saff, R.S. Varga, Zeros and poles of Pade approximants to exp(z), Numer. Math. 30 (1978) 241-266.

[17] W.C. Chew, J.M. Jin, E. Michielssen, Complex coordinate stretching as a generalized absorbing boundary condition, Microw. Opt. Technol. Lett. 15 (1997) 363-369.

[18] L. Knizhnerman, Personal communication.

[19] A. Vaziri Astaneh, M.N. Guddati, Efficient dispersion curve calculation for multilayered waveguides and half-spaces, Comput. Meth. Appl. Mech. Eng. 300 (2016) 27-46.

[20] F. Ihlenburg, Finite element analysis of acoustic scattering, Springer-Verlag, New York, NY, 1998.

[21] C. Pozrikidis, Introduction to finite and spectral element methods using MATLAB., 2nd ed., Chapman \& Hall CRC, Boca Raton, FL, 2014.

[22] A. Deraemaeker, I. Babuska, P. Bouillard, Dispersion and pollution of the FEM solution for the Helmholtz equation in one, two and three dimensions, Int. J. Numer. Methods Eng. 46 (1999) 471499.

[23] S. Asvadurov, V. Druskin, S. Moskow, Optimal grids for anisotropic problems, Electron. Trans. Numer. Anal. 26 (2007) 55-81. 